TRANSACTIONS OF THE

AMERICAN MATHEMATICAL SOCIETY

Volume 353, Number 4, Pages 1403-1425

S 0002-9947(99)02264-3

Article electronically published on April 23, 1999

\title{
REPRESENTABILITY IS NOT DECIDABLE FOR FINITE RELATION ALGEBRAS
}

\author{
ROBIN HIRSCH AND IAN HODKINSON
}

\begin{abstract}
We prove that there is no algorithm that decides whether a finite relation algebra is representable.

Representability of a finite relation algebra $\mathcal{A}$ is determined by playing a certain two player game $G(\mathcal{A})$ over 'atomic $\mathcal{A}$-networks'. It can be shown that the second player in this game has a winning strategy if and only if $\mathcal{A}$ is representable.

Let $\tau$ be a finite set of square tiles, where each edge of each tile has a colour. Suppose $\tau$ includes a special tile whose four edges are all the same colour, a colour not used by any other tile. The tiling problem we use is this: is it the case that for each tile $T \in \tau$ there is a tiling of the plane $\mathbb{Z} \times \mathbb{Z}$ using only tiles from $\tau$ in which edge colours of adjacent tiles match and with $T$ placed at $(0,0)$ ? It is not hard to show that this problem is undecidable.

From an instance of this tiling problem $\tau$, we construct a finite relation algebra $R A(\tau)$ and show that the second player has a winning strategy in $G(R A(\tau))$ if and only if $\tau$ is a yes-instance. This reduces the tiling problem to the representation problem and proves the latter's undecidability.
\end{abstract}

\section{INTRODUCTION}

In this paper we address an old question in algebraic logic: is there an algorithm that tells us whether or not a finite relation algebra is representable? We have not been able to pin down the origin of this problem precisely, but in all probability it originated with Roger Maddux. Maddux and McKenzie discussed it in the early 1980s, Maddux suggesting a solution by tiling (our approach here). It was raised again by McKenzie at a recent conference on universal algebra and lattice theory (Szeged, Hungary, 1996). The problem is listed in AMN91, page 730, open problem 3] (credited to Maddux). There is a discussion of the question in [Ma94 problems 13 and 14, page 463], where it is observed that the finite relation algebras can be partitioned into three classes: (a) the non-representable ones, (b) those that are representable over some finite set, and (c) the finite representable relation algebras with no representation over a finite set. It is not hard to show that (a) and (b)

Received by the editors April 2, 1997 and, in revised form, November 11, 1997.

1991 Mathematics Subject Classification. Primary 03G15; Secondary 03G05, 06E25, 03D35.

Key words and phrases. Relation algebra, representation, undecidability, tiling problem, games, algebraic logic.

Research of the second author was partially supported by UK EPSRC grant GR/K54946. Many thanks to Peter Jipsen, Maarten Marx, Szabolcs Mikulás, Mark Reynolds, Yde Venema and the referee for useful comments and for pointing out serious errors in early drafts of this paper. 
are recursively enumerable 1 The (isomorphism types of) finite relation algebras is clearly a recursive set. Consequently, (a), (b) and (c) are all recursive if and only if (c) is recursively enumerable. Maddux conjectures that the answer is no in both cases. In this paper we show that (a) is not recursive, thus confirming Maddux's conjecture. Hence the set of (isomorphism types of) finite representable relation algebras with no finite representation is not recursively enumerable. One problem remains open: is (b) recursive - i.e., given a finite relation algebra, is it decidable whether it has a representation over a finite set?

We will reduce the tiling problem to the question of whether a finite relation algebra is representable. That will show that the question is undecidable. This is utterly unsurprising, but our proof is rather complicated.

It is interesting to consider alternatives to this approach. For example, it has been known for some time that the representable relation algebras cannot be defined by a finite number of axioms [Mon64, and this alone suggests that for finite relation algebras the representability problem is undecidable. However, finite axiomatizability and decidability are not the same. If a class is finitely axiomatizable, then this does give us a decision method to test whether a finite object belongs to the class or not. But the converse is false: Németi shows that the relativized cylindric set algebras of any finite dimension greater than two are not finitely axiomatizable [HMT85, 5.5.12, 5.5.13, credited to Németi], yet it is decidable whether a finite structure is in this class or not [Nem96]. He further shows that the equational theory of this class is decidable.

One of the main motivations for Tarski's study of relation algebras was to define an alternative foundation for set theory. In [TG87] it is shown that relation algebras can act as a vehicle for set theory and hence all of mathematics. It would seem then, that undecidability results for relation algebra should be obtainable by this result. However, we have not been able to obtain the result of the present paper in that way.

Our construction originated in Hir95 and has been used in different forms in HH97a, Hod97.

We assume some familiarity with relation algebras. The uninitiated might try JT48, JT52, Ma91b, Ma91a, for example.

\section{Representability AND GAMES}

There have been a number of attempts to axiomatize the representable relation algebras (RRA), a key one being Tarski's axiomatization of the relation algebras [JT52, Definition 4.1]. This axiomatization turned out not to be complete Lyn50, Mon64 and Lyndon proposed a stronger, infinite axiomatization Lyn50] which we will refer to here as the Lyndon conditions. It turned out that the Lyndon conditions were not sound over RRA: there are representable relation algebras that fail some of the Lyndon conditions. Lyndon explained the error in his first axiomatization and produced a rather complex but correct axiomatization in Lyn56. Three separate axiomatizations of the closely related class of representable cylindric algebras appeared in HMT85, and alternative axiomatizations of representable relation and cylindric algebras appeared in $\mathrm{HH} 97 \mathrm{~b}$.

\footnotetext{
${ }^{1}$ Strictly, we have to consider the isomorphism type of members of these classes, so that we are dealing with sets and not classes.
} 
However, the Lyndon conditions do correctly characterize the representable relation algebras among the finite relation algebras; and in this paper, where we deal only with finite relation algebras, we will use a variant of these conditions to test representability.

In $[\mathrm{HH} 97 \mathrm{a}$ the Lyndon conditions are expressed in terms of a winning strategy for the second, 'existential player' in a certain two-player game, played over a relation algebra 2 Here we define a variant of these games that characterizes representability for finite relation algebras.

2.1. Atomic networks. Let $\mathcal{A}=\left(A,+,-, 0,1, ;,-, 1^{\prime}\right)$ be a finite relation algebra. We define an atomic network $N=(D, h)$ to consist of a finite set of nodes $D$ and a labelling function $h: D \times D \rightarrow A t(\mathcal{A})$, where $\operatorname{At}(\mathcal{A})$ is the set of atoms of $\mathcal{A}$, such that for all $d, e, f \in D$,

$$
\begin{aligned}
h(d, e) \leq 1^{\prime} & \Leftrightarrow d=e, \\
h(d, e) & =h(e, d)^{\smile}, \\
h(d, f) & \leq h(d, e) ; h(e, f) .
\end{aligned}
$$

In this paper we deal only with atomic networks, so we may refer to them simply as networks. Elsewhere a weaker definition is given for networks where only the right to left implication is required for the first rule. A network in which a two-way implication holds is called strict. However, in this paper all networks are assumed to be strict.

Let $M=(D, f), N=(E, g)$ be networks. If $D \supseteq E$ and $\left.f\right|_{E \times E}=g$, then we say that $M$ is an extension of $N$ and that $N$ is a subnetwork of $M$. We write $N \subseteq M$.

In the following we will use the same symbol $N$ to refer to the network, the set of nodes, and the labelling function, distinguishing the cases by their context. Thus, $n \in N$ means that $n$ is a node in the network $N$, and $N(m, n)$ stands for the label on the edge $(m, n) \in N \times N$.

\subsection{Games.}

Definition 1. Let $\mathcal{A}$ be a finite relation algebra, as before. $G(\mathcal{A})$ is a game of countable length in which two players, $\forall$ (male), and $\exists$ (female), build an increasing sequence of atomic networks

$$
N_{0} \subseteq N_{1} \subseteq N_{2} \subseteq \ldots
$$

In his first move, $\forall$ picks any atom $a \in A t(\mathcal{A})$, and $\exists$ responds with a network $N_{0}$ with nodes $m, n \in N_{0}$ such that $N_{0}(m, n)=a$. That completes the zero'th round of the play.

Suppose at the end of the $(k-1)$ th round that $\exists$ played the network $N_{k-1}(k>0)$. In the $k$ th round, $\forall$ picks any two nodes $m, n \in N_{k-1}$ and two atoms $a, b \in \operatorname{At}(\mathcal{A})$ such that $a ; b \geq N_{k-1}(m, n) . \exists$ responds with any network $N_{k} \supseteq N_{k-1}$ such that there is a node $p \in N_{k}$ with $N_{k}(m, p)=a$ and $N_{k}(p, n)=b$. That completes the $k$ th round.

\footnotetext{
${ }^{2}$ This simplified the conditions and showed a certain connection between the Lyndon conditions and the completely representable relation algebras — in fact, the Lyndon conditions axiomatize the theory of the completely representable relation algebras, though this class turns out not to be closed under elementary equivalence, so there are relation algebras that satisfy all the Lyndon conditions but have no complete representation.
} 
It is possible that in some round of the play $\exists$ is unable to make the required extension. In that case $\forall$ has won. If she does make a legal move in every round $k$ (for $k<\omega$ ), then she has won.

\section{Convention 1.}

1. There is no advantage to $\exists$ in adding more than a single node to the current network in each round of the play, and we assume throughout that she never does so, so that $\left|N_{k}\right| \leq\left|N_{k-1}\right|+1$ for all $k>0$.

2. If $\forall$ picks nodes $m, n \in N_{k-1}$ and atoms $a, b \in \mathcal{A}$, and if there is already a node $p \in N_{k-1}$ such that $N_{k-1}(m, p)=a$ and $N_{k-1}(p, n)=b$, then $\exists$ does not need to make a proper extension but can let $N_{k}=N_{k-1}$. As this kind of $\forall$-move is rather trivial, we will assume throughout that he never makes a move of this kind. With this assumption, $\exists$ is always forced to add a new point, and so $\left|N_{k}\right|=\left|N_{k-1}\right|+1$ for all $k>0$. If $\forall$ cannot make a move in some round, then $\exists$ wins straight away.

3. We regard $\forall$ as choosing the labels on the edges $(m, p),(p, m),(p, p),(p, n)$, and $(n, p)$, in the notation of Definition 1 above. (Of course, $(m, p)$ determines $(p, m)$, etc.) All other labels on edges of $N_{k}$ involving $p$ are regarded as having been chosen by $\exists$. This will be crucial later.

Theorem 1. Let $\mathcal{A}$ be a finite relation algebra. $\mathcal{A}$ is representable if and only if $\exists$ has a winning strategy in $G(\mathcal{A})$.

Proof. See, for example, [HH97b, Theorem 9] or [HH97a, Proposition 13]. The idea is essentially in [Lyn50] and is well known (e.g., [Ma82]).

\section{THE TILING PROBLEM}

An instance $\tau$ of the tiling problem is a finite set of square tiles $\tau=\left\{T_{0}, \ldots, T_{k-1}\right\}$. Each tile has a colour on each of its four edges: the four colours on the tile $T_{i}$ are $\operatorname{Top}\left(T_{i}\right), \operatorname{Bot}\left(T_{i}\right), \operatorname{Lt}\left(T_{i}\right)$ and $R t\left(T_{i}\right)$. See Figure 1. Note that the tiles have a fixed orientation.

Such an instance is said to be a yes-instance if it is possible to tile the plane $\mathbb{Z} \times \mathbb{Z}$. That is, there is a function $f:(\mathbb{Z} \times \mathbb{Z}) \rightarrow\{0, \ldots, k-1\}$ such that for all $x, y \in \mathbb{Z}$ we have $\operatorname{Lt}\left(T_{f(x+1, y)}\right)=\operatorname{Rt}\left(T_{f(x, y)}\right)$ and $\operatorname{Bot}\left(T_{f(x, y+1)}\right)=\operatorname{Top}\left(T_{f(x, y)}\right)$. We call such an $f$ a tiling. If there is no such tiling, then we have a no-instance.

The tiling problem (given an instance, is it a yes-instance or a no-instance?) is known to be undecidable [Ber66]. It is not hard to show from this that the following problem is also undecidable. Given a finite set of tiles $\left\{T_{0}, \ldots, T_{k-1}\right\}$ as above, is it the case that for each $i<k$ there is a tiling $f^{i}$ of the plane with $T_{i}$ placed at $(0,0)$ (formally $\left.f^{i}(0,0)=i\right)$ ?

We lose no generality if we assume that one tile, $T_{0}$, is a special tile such that all four edges have the same colour but this colour is not used on any edge of any other

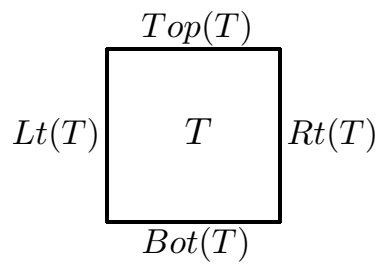

Figure 1. A tile $T$ 
tile. Thus, there is certainly a tiling $f^{0}$ with $f^{0}(0,0)=0$ given by $f^{0}(x, y)=0$ (all $x, y \in \mathbb{Z}$ ). $T_{0}$ can tile the plane on its own but not in combination with any of the other tiles. So given an arbitrary set of tiles $\left\{T_{1}, \ldots, T_{k-1}\right\}$, if we add the special tile, $T_{0}$, then $\left\{T_{1}, \ldots, T_{k-1}\right\}$ is a yes-instance if and only if the augmented set $\left\{T_{0}, \ldots, T_{k-1}\right\}$ is a yes-instance.

It is this version of the tiling problem that we use here.

Now, roughly, given an instance $\tau$ of the tiling problem we construct (by an algorithm) a finite relation algebra $R A(\tau)$ such that $\tau$ is a yes-instance if and only if $R A(\tau)$ is representable. Strictly, $R A(\tau)$ is what Maddux calls a weakly associative algebra (WA) Ma82, definition 1.2.4] - an algebra of the same type as a relation algebra satisfying all the Tarski axioms for relation algebras except, perhaps, the associativity axiom, but satisfying instead the weak associativity axiom

$$
\left(\left(1^{\prime} . x\right) ; 1\right) ; 1=\left(1^{\prime} . x\right) ;(1 ; 1) .
$$

$R A(\tau)$ is a relation algebra if and only if it is associative 3 We prove that

(i) if $R A(\tau)$ is associative (and so a relation algebra), then if it is representable, $\tau$ is a yes-instance (Theorems 1 and 3 );

(ii) if $\tau$ is a yes-instance, then $R A(\tau)$ is associative (Theorem 4 and Lemma 7), and representable (Theorems 1 and 4 ).

This suffices to prove the undecidability of the representation problem for finite relation algebras. To see this, note that the problem of whether a finite weaklyassociative algebra is associative is certainly decidable. If the representation problem for finite relation algebras were decidable, then given a tiling instance $\tau$, we could construct $R A(\tau)$ and decide if it is associative. If not, $\tau$ is a no-instance by (ii). If it is associative, then by (i,ii) it is a yes-instance if and only if $R A(\tau)$ is representable. Hence the tiling problem would be decidable, a contradiction.

\section{The Definition of $R A(\tau)$}

Notation. A representation of a relation algebra $\mathcal{A}$ is a map $X$ providing a binary relation $X(a)$ on some set, the domain of $X$, for each element $a$ of $\mathcal{A}$. Of course, $X$ respects the algebraic operations and is $1-1$.

In this section we define the algebra $R A(\tau)$. We will see (Lemma 2) that $R A(\tau)$ is a weakly associative algebra, but not integral $\|^{4}$ in fact, the identity $1^{\prime}$ is the disjunction of three units $e_{0}, e_{1}, e_{2}$. If $R A(\tau)$ is representable, then in any representation $X$, the domain $D$ of the representation will be the disjoint union of three subsets $-D=D_{0} \cup D_{1} \cup D_{2}$ - and for any point $d \in D$ and $i<3$ we have

$$
(d, d) \in X\left(e_{i}\right) \Leftrightarrow d \in D_{i} .
$$

As is standard for weakly associative algebras, every atom $a$ has a start unit $\operatorname{st}(a)=$ $1^{\prime} .(a ; \breve{a})$ and an end unit end $(a)=1^{\prime} .(\breve{a} ; a)$ [Ma82 Definition 5.10], which are atoms Ma82, Lemma 5.12.1]. Let $s t(a)=e_{i}$ and $\operatorname{end}(a)=e_{j}$ (some $i, j<3$ ). If $R A(\tau)$ has a representation $X$ with domain $D$, this tells us that for any pair of points $(e, d) \in X(a)$ we have $e \in D_{i}$ and $d \in D_{j}$. We will call such an atom an $(i-j)$-atom.

\footnotetext{
${ }^{3}$ It turns out that $R A(\tau)$ is associative if and only if the following condition is met: for each tile $T_{i} \in \tau$ there is a tile $T_{j} \in \tau$ such that $\operatorname{Rt}\left(T_{j}\right)=\operatorname{Lt}\left(T_{i}\right)$, along with three other conditions for the other sides of $T_{i}$.

${ }^{4} \mathrm{~A}$ suitable integral algebra $R A(\tau)$ can also be constructed, but we will not do so here.
} 
We will give the atoms of $R A(\tau)$ subscripts to indicate their start and end units. If the subscripts are equal, we generally write just one of them. E.g., $a_{01}, e_{22}=e_{2}$, etc.

If $n$ is any node in an $R A(\tau)$-network $N$, there is a unique unit $e_{i}$ such that $N(n, n)=e_{i}$ (some $i<3$ ). We will call such a node an $i$-node. If $n$ is an $i$-node and $m$ is a $j$-node of a network $N$, then the label $N(n, m)$ must be an $(i-j)$-atom. $(n, m)$ is called an $(i-j)$ edge.

We will often use subscripts to denote implicitly the kind of node we are talking about. For example, $n_{i} \in N$ is implicitly stated to be an $i$-node, $m_{1}$ and $m_{1}^{\prime}$ are 1-nodes, and so on.

4.1. The atoms. If $\tau$ is a tiling instance with $k$ tiles $T_{0}, \ldots, T_{k-1}$, then $R A(\tau)$ has $2 k+28$ atoms. They are

\begin{tabular}{|cc|l|}
\hline start & end & Atoms \\
\hline 0 & 0 & $e_{0}, w_{0}$ \\
0 & 1 & $g_{01}, u_{01}, v_{01}, w_{01}$ \\
0 & 2 & $g_{02}, u_{02}, v_{02}, w_{02}$ \\
1 & 1 & $e_{1},+1_{1},-1_{1}, w_{1}$ \\
2 & 2 & $e_{2},+1_{2},-1_{2}, w_{2}$ \\
1 & 2 & $T_{12}^{i}(i<k), w_{12}$ \\
\hline
\end{tabular}

plus the converses of the $(0-1),(0-2)$ and $(1-2)$ atoms. If $i, j<3, i \neq j$, and $a_{i j}$ is any $(i-j)$ atom, we write $a_{j i}$ for $\breve{a}_{i j}$. Thus, the converse of $g_{02}$ is $g_{20}$. We consider some of the atoms to be coloured; the atoms $g_{01}, g_{10}, g_{02}$, and $g_{20}$ are green, and the atoms $w_{0}, w_{1}, w_{2}, w_{01}, w_{02}, w_{12}$ and their converses are white.

4.2. The atom structure. To define $R A(\tau)$ it remains to define the operations of converse and composition on the atoms. The operations on arbitrary elements are then defined by distribution over disjunction; see [Lyn50. For converse, we have already defined the converse of atoms with distinct subscripts. All the rest are self-converse except the following: the converse of $+1_{1}$ is $-1_{1}$ and the converse of $+1_{2}$ is $-1_{2}$, and vice versa.

Now we define composition. We do this by listing the inconsistent triangles $(a, b, c)$ of atoms. This is defined to mean that $a ; b . \breve{c}=0$. Recall that the Peircean transforms of the triangle $(a, b, c)$ are $(b, c, a),(c, a, b),(\breve{a}, \breve{c}, \breve{b}),(\breve{c}, \breve{b}, \breve{a})$, and $(\breve{b}, \breve{a}, \breve{c})$. By the Peircean law in WAs, it follows from the inconsistency of $(a, b, c)$ that its Peircean transforms must also be inconsistent. The following triangles, plus all Peircean transforms of them, are defined to be inconsistent. First, any triangle where the indices do not match is inconsistent; e.g., $\left(x_{i j}, y_{k l}, a\right)$ and $\left(x_{j}, y_{k l}, a\right)$ are inconsistent if $j \neq k$, for any atom $a$. Second, a triangle $\left(e_{i}, x, y\right)$ is inconsistent unless $x=\breve{y}$. Third, the following are all inconsistent (the last column indicates the type of nodes in the triangle):

(1) $\left(g_{10}, g_{02}, w_{21}\right)$

$0,1,2$,

(2) $\left(T_{12}^{i}, T_{21}^{j},+1_{1}\right)$ any $i, j<k$, unless $\operatorname{Lt}\left(T_{i}\right)=R t\left(T_{j}\right)$

(3) $\left(u_{10}, g_{02}, T_{21}^{i}\right)$ any $i$ with $1 \leq i<k$

$0,1,2$,

(4) $\left(v_{10}, g_{01}, \pm 1_{1}\right)$

$0,1,1$.

There are three dual rules for inconsistent triangles, obtained from rules 2 3and 4 by swapping the subscripts 1 and 2 throughout and replacing $L t, R t$ by Bot, Top, 
respectively. We write $\pm 1_{1}$ as an abbreviation for 'either $+1_{1}$ or $-1_{1}$ '. We will refer to these inconsistent triangles by 'rules 1 to 4 .

All other triangles are defined to be consistent. This suffices to define composition. The resulting operation may not be associative (see [Lyn50, page 710]), but we will prove associativity later (Lemma 7), in the case that is important to us. Note that $1^{\prime}=e_{0}+e_{1}+e_{2}$ follows from this definition of consistency.

Clearly, we can obtain $R A(\tau)$ from $\tau$ effectively (by an algorithm).

Lemma 2. For any instance of the tiling problem $\tau, R A(\tau)$ is a weakly associative algebra.

Proof. Let $C$ be the set of consistent triangles of $R A(\tau)$. By Theorem 2.2.3 of Ma82 5 it suffices to show that

(i) $C$ is closed under Peircean transforms,

(ii) if $\left(e_{i}, x, y\right) \in C$, then $x=\breve{y}$ and

(iii) for any atom $a_{i j}$ of $R A(\tau)\left(e_{i}, a_{i j}, a_{j i}\right) \in C$.

This is rather easy to verify from the definition of the atom structure of $R A(\tau)$, bearing in mind that the only inconsistent triangles involving a unit $e_{i}$ are those where the indices do not match and those which break requirement (ii) above.

Remarks. It is helpful to think of the atoms $T_{12}^{i}$ as corresponding to the tiles $T_{i}(i<k)$. Because of this correspondence, we call an edge $\left(n_{1}, n_{2}\right)$ of a network $N$ a tile edge if $N\left(n_{1}, n_{2}\right)=T_{12}^{i}$ (some $i<k$ ). (Recall our convention that $n_{1}$ is a 1 -node here, so that $N\left(n_{1}, n_{1}\right)=e_{1}$, and similarly for $n_{2}$.) The atoms $T_{12}^{i}: i<k$ are called tile atoms.

Rule 2 (and its dual) force the tile edges to form a tiling pattern, as we will see in Theorem 3 ,

\section{WINNING STRATEGY IMPLIES TILING}

Theorem 3. Let $\tau$ be a set of tiles such that $T_{0}$ is a special tile with all four edges equal to each other but distinct from the colours used by any other tile. If $\exists$ has a winning strategy for $G(R A(\tau))$, then for each $i<k$ there is a tiling of the plane with $T_{i}$ at $(0,0)$, so $\tau$ is a yes-instance.

Proof. Suppose $\exists$ has a winning strategy and let $\forall$ make the following moves. First he plays the atom $T_{12}^{i}$, and in the next round he picks atoms $g_{10}, g_{02}$, so forcing the triangle in Figure 2(a) (in the diagram we have labelled $\left(c, a_{0}\right)$ with $g_{01}$ instead of labelling $\left(a_{0}, c\right)$ with $g_{10}$, but of course this is equivalent). Then he picks the edge $\left(b_{0}, c\right)$ and the two atoms $+1_{2}, g_{20}$, as in Figure 2(b).

$\exists$ has to fill in and label the missing edge $\left(a_{0}, b_{1}\right)$. She can do so because she has a winning strategy. The label must lie under $\left(g_{10} ; g_{02}\right) .\left(T_{12}^{i} ;+1_{2}\right)$. By rule 1 she cannot choose $w_{12}$, so the label must be $T_{12}^{j}$ for some $j<k$. By rule 2 she must choose $j$ so that $\operatorname{Bot}\left(T_{j}\right)=\operatorname{Top}\left(T_{i}\right)$.

$\forall$ continues with the two moves shown in Figure[3. In this way $\forall$ constructs two sequences of nodes $a_{0}, a_{1}, a_{2}, \ldots$ and $b_{0}, b_{1}, b_{2}, \ldots$ He can also extend the sequences backwards $\left(\ldots, a_{-1}, a_{0}, a_{1}, \ldots\right)$ by playing suitable moves. $\exists$ has to label each edge $\left(a_{x}, b_{y}\right): x, y \in \mathbb{Z}$ and in each case the only consistent choices are $T_{12}^{j}$ for some $j<k$.

It is fairly simple (by rule2) to see that this defines a tiling $f^{i}$ of the plane with $T_{i}$ at $(0,0)$. Formally, if the edge $\left(a_{x}, b_{y}\right)$ is labelled by $T_{12}^{j}$, then let $f^{i}(x, y)=j$.

\footnotetext{
${ }^{5}$ Maddux defines $(a, b, c)$ to be consistent iff $a ; b \geq c$ whereas here we mean that $a ; b \geq \breve{c}$.
} 


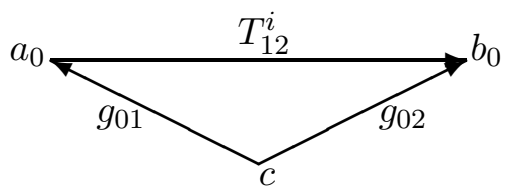

(a)

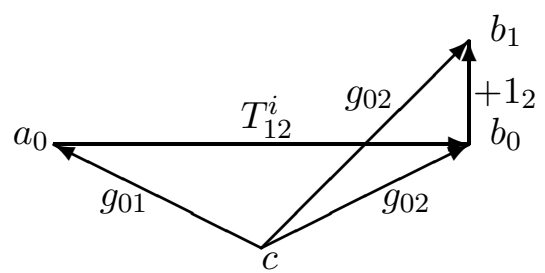

(b)

FIGURE 2.

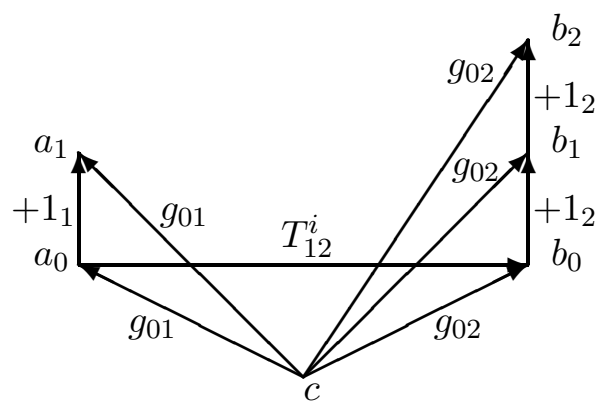

FiguRE 3. The play after five rounds

\section{Tiling IMPLies WinNing $\exists$-STRATEGY}

To complete the reduction of the tiling problem to the representation problem we need to prove the converse to Theorem 3

Theorem 4. Let $\tau$ be an instance of the tiling problem where each tile is part of a tiling of the plane, and one tile $T_{0} \in \tau$ is a special tile with all four edges the same colour, a colour not used by any other tile. Then $\exists$ has a winning strategy in the game $G(R A(\tau))$.

Proof. Assume the hypotheses. Let $f^{i}$ be a tiling function where $f^{i}(0,0)=$ $i$ (each $i<k$ ). We will provide a winning strategy for $\exists$ in the game $G(R A(\tau)$ ).

Initially, if $\forall$ plays the atom $a_{i j}$ of $R A(\tau)$, then $\exists$ responds with a network $N_{0}$ consisting only of the nodes $n_{i}, n_{j}$, equal if $a_{i j}$ is a unit and distinct if not, with $N_{0}\left(n_{i}, n_{j}\right)=a_{i j}, N_{0}\left(n_{j}, n_{i}\right)=a_{j i}, N_{0}\left(n_{i}, n_{i}\right)=e_{i}$, and $N_{0}\left(n_{j}, n_{j}\right)=e_{j}$. By the second part of the definition of consistency of triangles, this is clearly a well-defined network (in particular, if $a_{i j} \leq 1^{\prime}$, then $a_{i j}=e_{i}=e_{j}$ ).

Suppose, at some stage in the continuing play of $G(R A(\tau))$, that the current network is $N . \forall$ picks two nodes $n_{i}, n_{j} \in N$ and two atoms $a_{i l}, b_{l^{\prime} j}$ such that $a_{i l} ; b_{l^{\prime} j} \geq N\left(n_{i}, n_{j}\right)$. Necessarily, $l=l^{\prime}$. An $\forall$-move of this kind is called an $l$-move, and, bearing in mind Convention 1(2), it forces $\exists$ to add an $l$-node to $N$. She has to find a network $M$ extending $N$ containing a node $n_{l} \in M$ such that $M\left(n_{i}, n_{l}\right)=a_{i l}$ and $M\left(n_{l}, n_{j}\right)=b_{l j}$.

Convention 2. Throughout, if we define the labelling of an edge $M(p, q)=c \in$ $\operatorname{At}(\mathcal{A})$, then the labelling on the converse edge is implicitly defined by $M(q, p)=\breve{c}$. 
$\exists$ first builds a complete but partially labelled graph $N^{\forall}$ whose nodes are $N \cup$ $\left\{n_{l}\right\}$, where $n_{l}$ is a new $l$-node, not in $N$. (I.e. every pair of nodes in $N^{\forall}$ forms an edge, but not all edges are labelled.) $N^{\forall}$ extends $N$, so that if $p, q \in N$, then $N^{\forall}(p, q)=N(p, q)$. Also, $N^{\forall}\left(n_{i}, n_{l}\right)=a_{i l}, N^{\forall}\left(n_{l}, n_{j}\right)=b_{l j}$, and $N^{\forall}\left(n_{l}, n_{l}\right)=$ $e_{l}$. As with Convention 2, labels on the reverse edges $\left(n_{l}, n_{i}\right)$ and $\left(n_{j}, n_{l}\right)$ are now specified as well. (These are the edges we regard as being labelled by $\forall-$ recall Convention 1). It can be checked that this is well-defined if $n_{i}=n_{j}$. No other edges are labelled in $N^{\forall}$. Clearly, $N^{\forall}$ is consistent — for any three nodes $p, q, r \in N^{\forall}$, if all three edges of the triangle $(p, q, r)$ are labelled in $N^{\forall}$, then $N^{\forall}(p, r) \leq N^{\forall}(p, q) ; N^{\forall}(q, r)$, else $\forall$ has made an illegal move.

We now define a strategy for $\exists$ in choosing an atom for each unlabelled edge of $N^{\forall}$. Such edges have the form $\left(n_{l}, m\right)$ for $m \in N \backslash\left\{n_{i}, n_{j}\right\}$. Employing this strategy results in a completely labelled graph, say $M$.

We show that the strategy is winning for $\exists$ by showing that the graph $M$ is in fact an atomic network. In order to do this, we have to show for any three nodes $p, q, r \in$ $M$ that the triangle $(p, q, r)$ is consistent, i.e., that $M(p, r) \leq M(p, q) ; M(q, r)$. If all three edges $(p, r),(p, q)$ and $(q, r)$ are labelled in $N^{\forall}$, then we may assume that the triangle is consistent. If not, then if two of $p, q, r$ are equal, consistency is assured by Convention 2 and our definition of $M\left(n_{l}, n_{l}\right)=e_{l}$, so long as $\exists$ always uses an $i, j$-atom to label an $(i-j)$-edge (and she will). So it suffices to check consistency of the triangles with three distinct nodes and an edge labelled by $\exists$ in the current round of the game. We will do this as we define the strategy: for each edge unlabelled in $N^{\forall}$, we will explain which atom $\exists$ chooses to label it, and check that any triangle containing it conforms with rules 14 of the definition of $R A(\tau)$.

Remark 1. Since all this takes up what remains of the paper, it may help the reader if we discuss the underlying idea a little, before plunging in. The critical part of the strategy is where $\exists$ is forced to choose a $(1-2)$-atom - a tile atom or $w_{12}$ to label a $(1-2)$ edge. The atom $w_{12}$ is a sort of 'wild card' which may be adjacent to any tile as it is not mentioned in rule 2 So where possible, she chooses the atom $w_{12}$ to label such edges.

However, rule 1 prohibits the use of $w_{12}$ in some circumstances. When rule 1 applies, $\exists$ is forced to choose a tile atom. To help decide which one, we will assume that each tile edge in $N$ is associated with a genuine tiling of the plane, in the same way as happened in Theorem 3 This means that every tile edge $\left(n_{1}, n_{2}\right)$ in $N$ has an associated tiling function $f_{\left(n_{1}, n_{2}\right)} \in\left\{f^{0}, \ldots, f^{k-1}\right\}$, and sometimes also a pair of co-ordinates $\operatorname{Co}\left(n_{1}, n_{2}\right) \in \mathbb{Z} \times \mathbb{Z}$, so that the tile atom labelling $\left(n_{1}, n_{2}\right)$ is given by the tile $T_{f_{\left(n_{1}, n_{2}\right)}\left(C o\left(n_{1}, n_{2}\right)\right)}$ if $f_{\left(n_{1}, n_{2}\right)} \neq f^{0}$, and by $T_{0}$, otherwise. Except in $\forall$ 's current triangle, which is in $N^{\forall}$ so is assumed consistent, the tilings and co-ordinates will have to fit together in a coherent way rather as in Theorem 3

These tilings and co-ordinates will be assumed (inductively) to be given for $N$, and one of $\exists$ 's tasks will be to extend them to $M$. (The $u$ - and $v$-atoms play an important role here: the way her strategy deployed $v_{01}$ and $v_{02}$ in earlier rounds of the game will ensure that she can define tiling functions for new tile edges coherently, and $u_{01}, u_{02}$ do the same job for the co-ordinates.) When she has done this, it will be easy for her to decide which tile atom should label each new tile edge - she will just choose the one given by its tiling function and co-ordinates. Consistency of her choices will follow from the fact that the tiling function is a genuine tiling of the plane. 


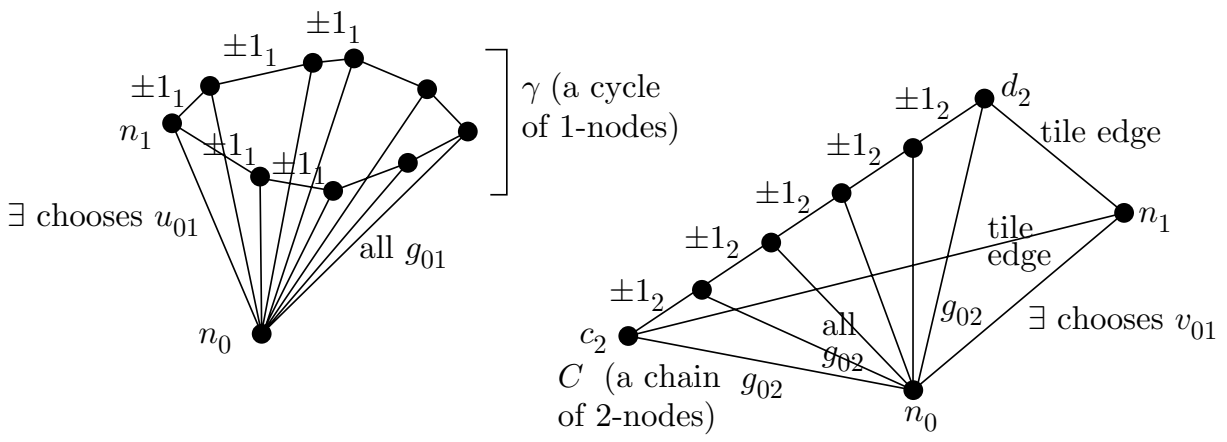

FIGURE 4. ヨ's strategy, case II $(1,2)$

The details of the tiling and co-ordinate functions will be discussed below, when we come to describe $\exists$ 's strategy for choosing tile atoms in full.

6.1. $\exists$ 's strategy. Here is $\exists$ 's strategy for labelling edges of $M$, unlabelled in $N^{\forall}$. We will define it so that:

( $\alpha) \exists$ never chooses a green atom, $\pm 1_{1}$, or $\pm 1_{2}$ to label an edge;

( $\boldsymbol{\beta})$ she never chooses a tile atom $M\left(n_{1}, n_{2}\right)=T_{12}^{i}$ unless there is a node $n_{0} \in N$ such that $N^{\forall}\left(n_{0}, n_{1}\right)=g_{01}$ and $N^{\forall}\left(n_{0}, n_{2}\right)=g_{02}$. In particular, she never chooses a tile atom unless one of the atoms chosen by $\forall$ in the current round of the game is green.

I: $(0-0),(1-1)$, and $(2-2)$ edges: For each of these types of edges she chooses the label $w_{i}$ for suitable $i$. Since $w_{i}$ is not mentioned in any of rules 1 to 4 , it follows that any triangle containing such an edge must be consistent.

II: $(0-1)$ and $(0-2)$ edges: We define the strategy for a (0-1) edge $\left(n_{0}, n_{1}\right)$; the strategy for $(0-2)$ edges is similar. $\exists$ always chooses either $u_{01}, v_{01}$ or $w_{01}$ for a $(0-1)$ edge.

1. Suppose there is a cycle of distinct 1-nodes of $N^{\forall}$, say $\gamma=\left\langle g^{0}, g^{1}, \ldots\right.$, $\left.g^{l-1}\right\rangle$ (some $l \geq 3$ ), such that

(a) $n_{1} \in \gamma$,

(b) $N^{\forall}\left(g^{i}, g^{i+1}\right)= \pm 1_{1}($ each $i<l-1)$, and $N^{\forall}\left(g^{l-1}, g^{0}\right)= \pm 1_{1}$,

(c) for all $g^{i} \in \gamma$, if $g^{i} \neq n_{1}$, then $N^{\forall}\left(n_{0}, g^{i}\right)=g_{01}$.

Then $\exists$ lets $M\left(n_{0}, n_{1}\right)=u_{01}$.

2. If there is a chain of 2 -nodes $C=\left\langle c_{2}, \ldots, d_{2}\right\rangle \subseteq N$, with $c_{2} \neq d_{2}$, such that

(a) for each $\alpha_{2} \in C, N^{\forall}\left(n_{0}, \alpha_{2}\right)=g_{02}$,

(b) each edge between two consecutive nodes in the chain $C$ is labelled by $\pm 1_{2}$ in $N$,

(c) $N^{\forall}\left(n_{1}, c_{2}\right)=T_{12}^{i}$ and $N^{\forall}\left(n_{1}, d_{2}\right)=T_{12}^{j}$ (some $i, j<k$ ),

then she lets $M\left(n_{0}, n_{1}\right)=v_{01}$.

3. Otherwise, she lets $M\left(n_{0}, n_{1}\right)=w_{01}$.

See Figure 4 .

We should check first that this strategy is well-defined - i.e., cases II(1) and $\mathrm{II}(2)$ do not apply simultaneously. So suppose both cases apply to the edge $\left(n_{0}, n_{1}\right)$. Since case $\mathrm{II}(1)$ applies, there is a cycle $\gamma$ as described above; and since case $\mathrm{II}(2)$ applies, there is a chain $C$ as above with endpoints $c_{2}$ 
and $d_{2}$. As the edge $\left(n_{0}, n_{1}\right)$ is just being labelled by $\exists$, either $n_{0}$ or $n_{1}$ is currently being added to the network. Now the new node, whichever it is, is incident with at most two labelled edges in $N^{\forall}$, the ones labelled by $\forall$ in the current round. But both $n_{0}$ and $n_{1}$ are incident with at least four such edges, because $N^{\forall}\left(n_{0}, g\right)=g_{01}\left(g \in \gamma \backslash\left\{n_{1}\right\}\right)$ and $N^{\forall}\left(n_{0}, \alpha_{2}\right)=g_{02}\left(\alpha_{2} \in C\right)$, while $N^{\forall}\left(n_{1}, c_{2}\right), N^{\forall}\left(n_{1}, d_{2}\right)$ are labelled in $N^{\forall}$ (they are tile atoms) and the two edges connecting $n_{1}$ to its neighbours in $\gamma$ are also labelled (with $\pm 1_{1}$ ) in $N^{\forall}$. This is a contradiction, and shows that the strategy is well-defined.

Next we check that it is consistent: when the labelling of all edges of $M$ has been completed, no triangle containing an edge labelled by case II of the strategy is inconsistent. Though we have not yet described the rest of the strategy, so we don't know exactly how edges of $M$ are labelled, we promise that conditions $(\boldsymbol{\alpha})$ and $(\boldsymbol{\beta})$ will in the end be met, and these are all we need.

First, we check that if $\exists$ chooses the atom $u_{01}$ for the edge $\left(n_{0}, n_{1}\right)$, then there is no 2 -node $n_{2}$ such that the triangle $\left(n_{0}, n_{1}, n_{2}\right)$ violates rule 3 in $M$. Suppose this happened: so $M\left(n_{0}, n_{1}\right)=u_{01}, M\left(n_{0}, n_{2}\right)=g_{02}$, and $M\left(n_{1}, n_{2}\right)=T_{12}^{i}$ for some $i$ with $1 \leq i<k$. (Recall that rule 3 does not apply to $T_{12}^{0}$.) Now $n_{0}$ is incident with at least three green edges - two labelled $g_{01}$ into the cycle $\gamma$ that caused the use of $u_{01}$ and one labelled $g_{02}$ to $n_{2}$ so by $(\boldsymbol{\alpha}), n_{1}$ must be the node added in the current round. $n_{1}$ is incident with two edges labelled $\pm 1_{1}$ in the cycle $\gamma$, so by $(\boldsymbol{\alpha})$, these are the two edges chosen by $\forall$ in the current round. But then, as neither of $\forall$ 's atoms is green, by $(\boldsymbol{\beta}) \exists$ would not have chosen a tile atom for $M\left(n_{1}, n_{2}\right)$. Thus we have a contradiction. So if $\exists$ chooses $M\left(n_{0}, n_{1}\right)=u_{01}$, then any triangle involving this edge is consistent.

The second possible inconsistency that we have to check for is when $\exists$ chooses $v_{01}$ for the edge $\left(n_{0}, n_{1}\right)$, and there is a 1-node $m_{1}$ such that the triangle $\left(n_{0}, n_{1}, m_{1}\right)$ violates rule 4 Suppose there is a chain $C$ with endpoints $c_{2} \neq d_{2}$, with $N^{\forall}\left(n_{1}, c_{2}\right)$ and $N^{\forall}\left(n_{1}, d_{2}\right)$ both tile atoms, as in case II(2) of the strategy, and with $N^{\forall}\left(n_{0}, m_{1}\right)=g_{01}$ and $N^{\forall}\left(m_{1}, n_{1}\right)= \pm 1_{1}$. As before, because the labels $g_{02}$ on the edges $\left(n_{0}, c_{2}\right)$ and $\left(n_{0}, d_{2}\right)$ must have been chosen by $\forall$, this could only happen if $n_{1}$ is the node currently added, as $c_{2} \neq d_{2}$. Now the edges $\left(n_{1}, c_{2}\right)$ and $\left(n_{1}, d_{2}\right)$ are in $N^{\forall}$, and as this exhausts the labelled $N^{\forall}$-edges incident with $n_{1}$, the edge $\left(n_{1}, m_{1}\right)$ must be labelled by $\exists$ in the current round. This conflicts with $(\boldsymbol{\alpha})$, because $\exists$ never uses $\pm 1_{1}$ to label an edge. Thus, the situation described does not arise.

Hence, if this strategy is used, any triangle in $M$ involving a $(0-1)$ edge labelled by $\exists$ must be consistent. Similarly, triangles involving $(0-2)$ edges chosen by $\exists$ are consistent.

Having dealt with all $(0-1),(0-2),(1-1)$ and $(2-2)$ edges now, we can see that $(\boldsymbol{\alpha})$ is indeed a true property of $\exists$ 's strategy.

III: $(1-2)$ non-tile edges: $\exists$ now proceeds to deal with the new $(1-2)$ edges not occurring in $N^{\forall}$. Let $\left(n_{1}, n_{2}\right)$ be such an edge. If it is consistent with $N^{\forall}$, she lets $M\left(n_{1}, n_{2}\right)=w_{12}$. In more detail, if there is no node $m_{0} \in N$ such that $N^{\forall}\left(m_{0}, n_{1}\right)=g_{01}$ and $N^{\forall}\left(m_{0}, n_{2}\right)=g_{02}$, then she lets $M\left(n_{1}, n_{2}\right)=w_{12}$. Such an edge cannot be part of an inconsistent triangle in $M$, because the only inconsistent triangles involving the atom $w_{12}$ are those mentioned in rule 1 and because $\exists$ never chooses green atoms. The edges so labelled are not tile edges. 
This means that $(\boldsymbol{\beta})$ is also a true property of $\exists$ 's strategy.

IV: $(1-2)$ tile edges: Finally, $\exists$ has to label the remaining $(1-2)$ edges of $M$, if any. These are the edges of the form $\left(n_{1}, n_{2}\right)$ such that for some node $m_{0} \in N, N^{\forall}\left(m_{0}, n_{1}\right)=g_{01}$ and $N^{\forall}\left(m_{0}, n_{2}\right)=g_{02} \cdot \exists$ is not allowed to choose the atom $w_{12}$ for $\left(n_{1}, n_{2}\right)$ because of rule 1 - she must use a genuine tile atom here.

To choose tile atoms for these edges, $\exists$ will take advantage of certain tilings and (possibly) co-ordinates associated with existing tile edges; and to continue winning later on, she will also have to extend these tilings and co-ordinates to the new tile edges constructed in the current round. This includes any new tile edges labelled by $\forall$, so that $\exists$ may have to define tilings/co-ordinates even if there are no new tile edges for her to label.

These tilings and co-ordinates will be assumed inductively to comply with the conditions T1, T2 and T3 below. To specify these, we need to define some terms.

Definition 2. Let $X \in\{N, M\}$.

- Let $p, q, r$ be distinct nodes of $X$. The triangle $(p, q, r)$ is said to be an $\forall$ triangle if it was constructed by $\forall$ in some round of the game. More formally, suppose (without loss) that node $r$ was the most recently constructed node out of $p, q, r$ as the game progressed. Then triangle $(p, q, r)$ is an $\forall$-triangle if in the round when $r$ was added, $\forall$ chose $p, q$ as his nodes (and $X(p, r), X(r, q)$ as his atoms). The order of the nodes $p, q, r$ is not significant here, so if $(p, q, r)$ is an $\forall$-triangle, then so are $(q, r, p)$ and $(q, p, r)$.

- Recall that a tile edge is one labelled with $T_{12}^{i}$ for some $i<k$. Two edges $\left(m_{1}, m_{2}\right)$ and $\left(m_{1}, m_{2}^{\prime}\right)$ of $X$ are said to be attached to each other if they are both tile edges, $N\left(m_{2}, m_{2}^{\prime}\right)= \pm 1_{2}$, and the triangle $\left(m_{1}, m_{2}, m_{2}^{\prime}\right)$ is not an $\forall$ triangle. Similarly, if $\left(m_{1}, m_{2}\right)$ and $\left(m_{1}^{\prime}, m_{2}\right)$ are both tile edges, $N\left(m_{1}, m_{1}^{\prime}\right)=$ $\pm 1_{1}$, and the triangle $\left(m_{1}, m_{1}^{\prime}, m_{2}\right)$ is not an $\forall$-triangle, then the two edges are attached.

In a nutshell, two tile edges are attached if they form two sides of a non$\forall$-triangle, the third side of which is labelled by a \pm 1 atom.

In this definition, we are not concerned with the orientation of the edges - we regard them as undirected edges.

- Two tile edges $\left(m_{1}, m_{2}\right)$ and $\left(m_{1}^{\prime}, m_{2}^{\prime}\right)$ are said to be linked in $X$ if and only if they are equal or there is a chain of tile edges in $X$ from $\left(m_{1}, m_{2}\right)$ to $\left(m_{1}^{\prime}, m_{2}^{\prime}\right)$ with each edge in the chain attached to the next one. Thus, 'linked' is the reflexive, transitive closure in $X$ of the 'attachment' relation. It is an equivalence relation on tile edges.

At this stage $\exists$ has labelled all the edges of $M$ except the $(1-2)$ tile edges. Although she has not yet labelled these, she knows which edges are going to be tile edges (by $(\boldsymbol{\beta}))$, and she knows the labels on all $(1-1)$ and $(2-2)$ edges. She also knows which triangles $\forall$ has picked during the game, of course. Therefore it makes sense to say that two tile edges of $M$ are attached or linked to each other in $M$.

6.2. Tiling and co-ordinate requirements for $N$. We require (inductively) that each tile edge $\left(n_{1}, n_{2}\right)$ of $N$ - whether its label was chosen by $\exists$ or $\forall$ - is associated with a tiling $f=f_{\left(n_{1}, n_{2}\right)} \in\left\{f^{i}: i<k\right\}$ in such a way that

T1: if the tile edges $\left(n_{1}, n_{2}\right),\left(n_{1}^{\prime}, n_{2}^{\prime}\right)$ are linked in $N$, then $f_{\left(n_{1}, n_{2}\right)}=f_{\left(n_{1}^{\prime}, n_{2}^{\prime}\right)}$. 
If $f=f^{0}$, we do not need co-ordinates. (Recall that all four edges of the tile $T_{0}$ have the same colour.) If $f \neq f^{0}$, we need co-ordinates $\operatorname{Co}\left(n_{1}, n_{2}\right)=(x, y)$, say, where $x, y \in \mathbb{Z}$, such that:

T2: If $\left(n_{1}, n_{2}^{\prime}\right)$ is attached to $\left(n_{1}, n_{2}\right), f_{\left(n_{1}, n_{2}\right)}=f_{\left(n_{1}, n_{2}^{\prime}\right)} \neq f^{0}, N\left(n_{2}, n_{2}^{\prime}\right)=+1_{2}$, and $\operatorname{Co}\left(n_{1}, n_{2}\right)=(x, y)$, then $\operatorname{Co}\left(n_{1}, n_{2}^{\prime}\right)=(x, y+1)$.

Similarly, if $\left(n_{1}^{\prime}, n_{2}\right)$ is attached to $\left(n_{1}, n_{2}\right)$, the associated tiling is not $f^{0}$, $N\left(n_{1}, n_{1}^{\prime}\right)=+1_{1}$, and $C o\left(n_{1}, n_{2}\right)=(x, y)$, then $C o\left(n_{1}^{\prime}, n_{2}\right)=(x+1, y)$.

T3: For each tile edge $\left(n_{1}, n_{2}\right)$, if $f_{\left(n_{1}, n_{2}\right)}=f^{0}$, then $N\left(n_{1}, n_{2}\right)=T_{12}^{0}$. If $f_{\left(n_{1}, n_{2}\right)}=f^{i}(i>0)$ and $C o\left(n_{1}, n_{2}\right)=(x, y)$, then $N\left(n_{1}, n_{2}\right)=T_{12}^{f^{i}(x, y)}$.

It is easy to arrange that the requirements T1, T2, and T3 hold in the initial network $N_{0}$. It will involve at most one tile edge (at most one edge of any kind!), and if its label is $T_{12}^{i}$, we let the associated tiling be $f^{i}$, and if $i>0$ we assign the co-ordinates $(0,0)$.

Now assume inductively that tilings and co-ordinates are defined for $N$, satisfying T1-T3. First, we will describe how to extend them to $M$. Then, when all tilings and co-ordinates have been defined, we will check that $\mathrm{T} 1$ and $\mathrm{T} 2$ are satisfied. Finally, T3 will tell $\exists$ which tile to use for each new tile edge, and then we can check consistency of triangles involving them.

6.3. Tiling functions and co-ordinates for $\forall$ 's tile edges. The first step is to define tiling functions and co-ordinates for any tile edges chosen by $\forall$ in the current round. If $\forall$ chooses $N^{\forall}\left(n_{1}, n_{2}\right)=T_{12}^{j}$, say, then let $f_{\left(n_{1}, n_{2}\right)}=f^{j}$, and if $j>0$ let $\operatorname{Co}\left(n_{1}, n_{2}\right)=(0,0)$, in agreement with T3.

6.4. Tiling functions for $\exists$ 's new tile edges. $\exists$ must now find tilings and perhaps co-ordinates for the new tile edges in $M$, if any. Suppose that there are some. Suppose also that the new node is a 2-node: i.e., the nodes of $M$ are those of $N$ plus a new node $n_{2} \notin N$. The other case, where the new node is a 1-node, is dealt with similarly, using the $(1-2)$ symmetry of the rules 14 defining the atom structure. This $n_{2}$ will be fixed in the notation from now on. The new tile edges are precisely those of the form $\left(n_{1}, n_{2}\right)$, where $n_{1} \in N$ and $N\left(m_{0}, n_{1}\right)=g_{01}, N^{\forall}\left(m_{0}, n_{2}\right)=g_{02}$ for some $m_{0} \in N . \exists$ has to associate a tiling function $f_{\left(n_{1}, n_{2}\right)}$ with each such edge. She does this as follows:

- If $\left(n_{1}, n_{2}\right)$ is linked (in $M$ ) to a tile edge $t$ of $N^{\forall}$, then she associates with $\left(n_{1}, n_{2}\right)$ whatever tiling function is associated with $t$. That is, she sets $f_{\left(n_{1}, n_{2}\right)}:=f_{(t)}$.

- Otherwise, she lets $f_{\left(n_{1}, n_{2}\right)}=f^{0}$.

Of course, we have to show that this is well-defined. This is done by the following lemma.

Lemma 5. Under the above assumptions (in particular, that $n_{2}$ is the new node and $\left(n_{1}, n_{2}\right)$ a tile edge to be labelled by $\exists$, so that $N^{\forall}\left(m_{0}, n_{2}\right)=g_{02}$ and $N\left(m_{0}, n_{1}\right)$ $=g_{01}$ for some $\left.m_{0} \in N\right)$ :

1. If $\left(n_{1}, n_{2}\right)$ is linked in $M$ to a tile edge of $N^{\forall}$ that is not in $N$, then $\left(n_{1}, n_{2}\right)$ is not linked in $M$ to any other tile edge of $N^{\forall}$.

2. Let $t, t^{\prime}$ be tile edges of $N$. If $t$ and $t^{\prime}$ are linked in $M$, then they are linked in $N$.

For suppose that $\left(n_{1}, n_{2}\right)$ is linked in $M$ to two distinct tile edges $t, t^{\prime}$ of $N^{\forall}$. By (1) of the lemma, $t, t^{\prime}$ are edges of $N$. Because 'linked' is an equivalence relation 


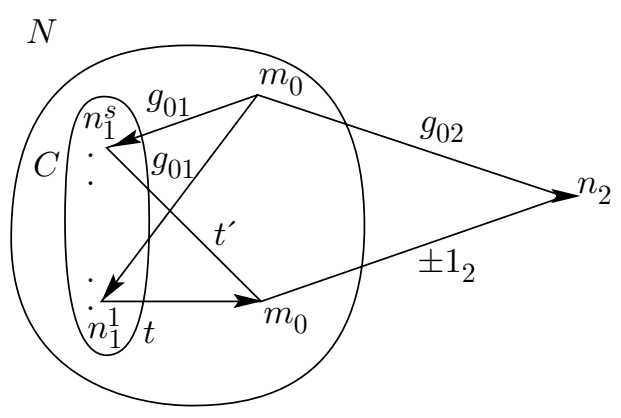

Figure 5. The chain linking $t$ to $t^{\prime}$

on tile edges, $t, t^{\prime}$ are linked in $M$. By (2) of the lemma, they are linked in $N$, so by T1 for $N, f_{(t)}=f_{\left(t^{\prime}\right)}$. Thus, $\exists$ 's definition of $f_{\left(n_{1}, n_{2}\right)}:=f_{(t)}$ is well-defined.

Proof. (1) Assume the hypotheses. Then $\forall$ 's chosen atoms in the current round must have been $g_{02}$ and a tile atom. Neither of them was $\pm 1_{2}$.

Suppose that $\left(n_{1}, n_{2}\right)$ are linked to a tile edge $t$ in $N$. Then there is a chain of attached tile edges proceeding from $\left(n_{1}, n_{2}\right)$ to $t$. At some stage, this chain crosses into $N$. This is impossible unless some edge of $M$ incident with $n_{2}$ is labelled $\pm 1_{2}$. But $\forall$ did not choose $\pm 1_{2}$, and $\exists$ never does (by $(\boldsymbol{\alpha})$ ). So $\left(n_{1}, n_{2}\right)$ is not linked to any tile edge of $N$. As there is a unique tile edge of $N^{\forall}$ that is not in $N$, this proves part (1).

(2) Assume not. Take a counterexample pair of tile edges $t, t^{\prime}$ of $N$, linked in $M$ but not in $N$, with the shortest possible chain of attached tile edges $e_{i}(1 \leq i \leq s)$ of $M$ linking $t$ with $t^{\prime}$ : so $t$ is attached to $e_{1}, e_{1}$ to $e_{2}, \ldots, e_{s-1}$ to $e_{s}$, and $e_{s}$ to $t^{\prime}$, and $s \geq 1$ is least possible. Clearly, no $e_{i}$ is an edge of $N$, or a counterexample with a shorter chain would be possible. Also, the $e_{i}$ are all distinct. So the $e_{i}$ have the form $\left(n_{1}^{i}, n_{2}\right)$ for distinct nodes $n_{1}^{i} \in N(1 \leq i \leq s)$. Since $t, t^{\prime}$ are edges of $N$, we must have $t=\left(n_{1}^{1}, m_{2}\right)$ and $t^{\prime}=\left(n_{1}^{s}, m_{2}^{\prime}\right)$ for some 2-nodes $m_{2}, m_{2}^{\prime} \in N$ with $M\left(n_{2}, m_{2}\right)= \pm 1_{2}$ and $M\left(n_{2}, m_{2}^{\prime}\right)= \pm 1_{2}$. Hence $\left(n_{2}, m_{2}\right),\left(n_{2}, m_{2}^{\prime}\right)$ are edges of $N^{\forall}$, chosen by $\forall$ in the current round. Now by the assumption of the lemma, $\forall$ also chose a green atom: $N^{\forall}\left(m_{0}, n_{2}\right)=g_{02}$. Because he only chose two edges, we must have $m_{2}=m_{2}^{\prime}$. Because $t \neq t^{\prime}$, we see that $n_{1}^{1} \neq n_{1}^{s}$, so $s \geq 2$. As $\forall \operatorname{did}$ not choose a tile atom in this round, the tile edges $\left(n_{1}^{i}, n_{2}\right)$ are going to be labelled by $\exists$, so by $(\boldsymbol{\beta})$ we must have $N\left(m_{0}, n_{1}^{i}\right)=g_{01}$ for all $i$. By considering the different labels on the edges $\left(x, n_{2}\right)$ for $x \in\left\{m_{0}, m_{2}, n_{1}^{i}: 1 \leq i \leq s\right\}$, we see that $m_{0}, m_{2}$, and the $n_{1}^{i}$ are all distinct. See Figure 5 .

We claim that $N\left(m_{0}, m_{2}\right)=g_{02}$. Given the claim, we have:

1. the edges $\left(n_{1}^{i}, m_{2}\right)$ are all tile edges of $N$ (by rule 1 since $N\left(m_{0}, n_{1}^{i}\right)=g_{01}$ for each $i)$.

2. for each $1 \leq i<s,\left(n_{1}^{i}, m_{2}\right)$ is attached to $\left(n_{1}^{i+1}, m_{2}\right)$.

For if not, $\left(n_{1}^{i}, n_{1}^{i+1}, m_{2}\right)$ must be an $\forall$-triangle: two sides of it were chosen by $\forall$ when its last node was constructed. So if $m_{0}$ was already in existence when this triangle was completed, the label on the edge from the last node of the triangle to $m_{0}$ must have been chosen by $\exists$. But this last node is connected to $m_{0}$ by a green edge (we use the claim again here in the case where the last node was $m_{2}$ ), and $\exists$ never chooses green labels. So $m_{0}$ must have been 


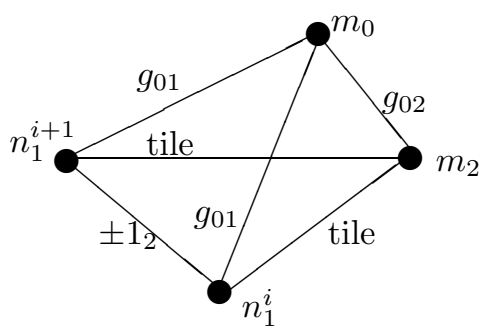

Figure 6. Successive tile edges are attached

constructed after the triangle $\left(n_{1}^{i}, n_{1}^{i+1}, m_{2}\right)$. This is also impossible, since it is connected to all three nodes of the triangle by green edges and at least one of these edges must have been labelled by $\exists$. See Figure 6

3. Hence $t$ is linked to $t^{\prime}$ in $N$, via the chain $t=\left(n_{1}^{1}, m_{2}\right),\left(n_{1}^{2}, m_{2}\right), \ldots$, $\left(n_{1}^{s}, m_{2}\right)=t^{\prime}$. This is what we wanted to show.

To prove the claim, suppose for a contradiction that $N\left(m_{0}, m_{2}\right) \neq g_{02}$. We have a chain of 1-nodes

$$
C=\left\langle n_{1}^{1}, \ldots, n_{1}^{s}\right\rangle \subseteq N
$$

such that $N\left(m_{0}, n_{1}^{i}\right)=g_{01}$ for all $n_{1}^{i} \in C$ and consecutive nodes in the chain are connected by $\pm 1_{1}$ (Figure 5). We know that the chain $C$ has distinct endpoints: $n_{1}^{1} \neq n_{1}^{s} \in C$. Which node out of $C \cup\left\{m_{0}, m_{2}\right\}$ was most recently added? The interior nodes $n_{1}^{2}, \ldots, n_{1}^{s-1}$ of $C$, if any, are incident with at least three edges within $C \cup\left\{m_{0}, m_{2}\right\}$ chosen by $\forall$ (consider green atoms and \pm 1 , as usual) and therefore none of these could be the most recently added node. The endpoints of $C, n_{1}^{1}$ and $n_{1}^{s}$, are each incident with one edge labelled $\pm 1_{1}$ and another labelled $g_{01}$. If $n_{1}^{1}$ (or $\left.n_{1}^{s}\right)$ was the most recently added, then, as $N\left(m_{0}, m_{2}\right) \neq g_{02}$ by assumption, we cannot account for the fact that $N\left(n_{1}^{1}, m_{2}\right)$ (respectively, $N\left(n_{1}^{s}, m_{2}\right)$ ) is a tile atom. So this can't happen. Thus, the most recently added node must be $m_{0}$ or $m_{2}$.

If $\exists$ chose the edge $\left(m_{0}, m_{2}\right)$ then, by her strategy for $(1-2)$ edges and because of the prior existence of the chain $C$, she would have chosen $N\left(m_{0}, m_{2}\right)=v_{02}$. But then, in the current round, $\forall$ 's move must be illegal, as $g_{02} ; \pm 1_{2} \nsucceq v_{02}$ (rule 4). So this can't happen, and it must be $\forall$ who chose the edge $\left(m_{0}, m_{2}\right)$. Hence, the most recently added node was, in fact $m_{2}$, for $m_{0}$ is incident with at least three edges, those to $m_{2}, n_{1}^{1}, n_{1}^{s}$, chosen by $\forall$.

We are supposing that $\left(m_{0}, m_{2}\right)$ is not green and we know now that this edge was labelled by $\forall$. Therefore, at least one of the two tile edges $\left(n_{1}^{1}, m_{2}\right),\left(n_{1}^{s}, m_{2}\right)$ was labelled by $\exists$. So by $(\boldsymbol{\beta}), \forall$ must have chosen the edge $N\left(n_{0}, m_{2}\right)=g_{02}$, for some $n_{0} \in N$. Since $\left(n_{0}, m_{2}\right)$ is green and $\left(m_{0}, m_{2}\right)$ is not, we have $n_{0} \neq m_{0}$. Hence $\forall$ 's two edges were $\left(m_{0}, m_{2}\right)$ and $\left(n_{0}, m_{2}\right)$. He chose neither of the tile edges $\left(n_{1}^{1}, m_{2}\right),\left(n_{1}^{s}, m_{2}\right)$ : both were chosen by $\exists$, and for her to do this we must have $N\left(n_{0}, n_{1}^{1}\right)=N\left(n_{0}, n_{1}^{s}\right)=g_{01}$. See Figure [

But now we consider which node from $C \cup\left\{m_{0}, n_{0}\right\}$ was most recently added. The interior nodes of $C$ are incident with three edges chosen by $\forall$ : two edges within $C$ labelled $\pm 1_{1}$, and one to $m_{0}$, labelled $g_{10}$, so it was not any of those. Nor was it 


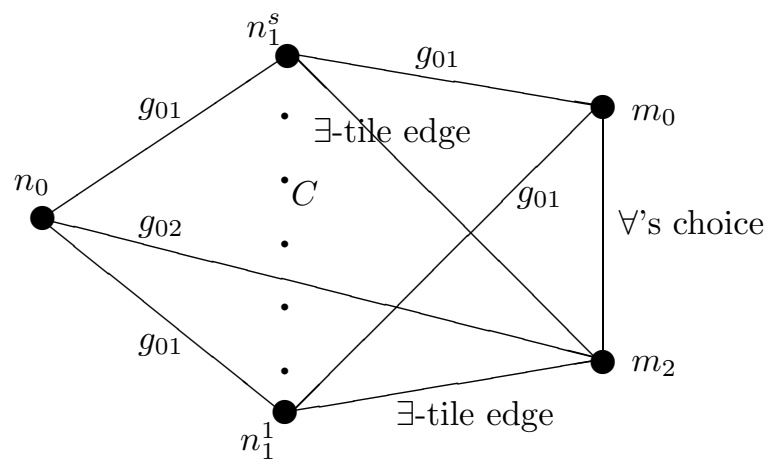

Figure 7 . The node $n_{0}$

either of the end nodes $n_{1}^{1}, n_{1}^{s}$, since they are each incident with a $\pm 1_{1}$ edge within $C$ and two green edges, to $n_{0}$ and $m_{0}$. So it must have been either $n_{0}$ or $m_{0}$. These are both connected to $n_{1}^{1}$ and $n_{1}^{s}$ by edges labelled by $g_{01}$, so in this round, $\forall$ must have chosen the nodes $n_{1}^{1}, n_{1}^{s}$ (recall that these are distinct) and the atoms $g_{10}, g_{01}$.

But this is in contravention to Convention 1, since there was a suitable node (namely whichever of $n_{0}, m_{0}$ already existed) in $N$ already, so $\forall$ would not have made such a move. This is a contradiction and completes the proof of the claim.

Now we have a well-defined association of tiling functions to all new tile edges not in $N$.

6.5. Co-ordinates. Next, we have to assign co-ordinates $C o\left(n_{1}, n_{2}\right)$ to each new tile edge $\left(n_{1}, n_{2}\right)$ of $M$ which is not an edge of $N^{\forall}$ and with $f_{\left(n_{1}, n_{2}\right)}=f^{i}$ for some $i>0$.

If there are no such edges, there is nothing to do. So assume that there is at least one; let $\left(n_{1}, n_{2}\right)$ be such an edge. Now, $i>0$ means that $\left(n_{1}, n_{2}\right)$ is linked to a tile edge of $N^{\forall}$ - either an edge of $N$, in which case $\forall$ 's second atom must be $\pm 1_{2}$, or a tile edge chosen by $\forall$ in the current round, in which case $\forall$ 's second atom is a tile atom (cf. the proof of Lemma 5 (1)). Either way, we see that in the current round, $\forall$ chose exactly one green atom - say, $N^{\forall}\left(m_{0}, n_{2}\right)=g_{02}$, for some unique $m_{0} \in N$. Like $n_{2}$, this $m_{0}$ will be fixed in our notation from now on.

Let

$$
\Gamma=\left\{n_{1} \in N: N\left(m_{0}, n_{1}\right)=g_{01}\right\} .
$$

$\exists$ has to give co-ordinates to each of the edges $\left(n_{1}, n_{2}\right)$, for $n_{1} \in \Gamma$, if its tiling function is not $f^{0}$. Define a set of 1 -nodes $\Gamma^{+}$by

- $\Gamma^{+}=\Gamma$ if $\forall$ 's second atom is $\pm 1_{2}$,

- $\Gamma^{+}=\Gamma \cup\left\{m_{1}\right\}$ if $\forall$ 's second atom is $N^{\forall}\left(m_{1}, n_{2}\right)=T_{12}^{j}$ (some necessarily unique $m_{1} \in N$ and $j<k$ ).

The tile edges incident with $n_{2}$ in $M$ are $\left\{\left(n_{1}, n_{2}\right): n_{1} \in \Gamma^{+}\right\}$.

Define a graph $G$ with nodes $\Gamma^{+}$and with an edge connecting two nodes $a, b$ of $G$ if and only if $N(a, b)= \pm 1_{1}$.

Lemma 6. The graph $G$ is acyclic. 
Proof. First, suppose that $\forall$ 's second atom is $\pm 1_{2}$, so that $\Gamma^{+}=\Gamma$. Assume for contradiction that there is a cycle in $G$ - say $\gamma=\left\langle g_{0}, \ldots g_{t-1}\right\rangle \subseteq G$ (some $t \geq 3$ ) with graph edges of $G$ connecting $g_{0}$ to $g_{1}, g_{1}$ to $g_{2}, \ldots, g_{t-1}$ to $g_{0}$. We ask the question: in the course of the game, which node of $\gamma \cup\left\{m_{0}\right\}$ was most recently added to $N$ ? Whichever it is, it can be connected to the rest of $\gamma \cup\left\{m_{0}\right\}$ by at most two edges labelled by $\forall$. Now each node in $\gamma$ is incident with two edges labelled $\pm 1_{1}$ (because it is in the cycle $\gamma$ ) and one green edge connecting it to $m_{0}$ (because it is in $\Gamma$ ), and all of these edges are only chosen by $\forall$. Thus, none of these can have been the most recently added node, and the last-added node must be $m_{0}$. But $|\gamma| \geq 3$, and $m_{0}$ is connected to every node of $\gamma$ by a green edge. As before, this is impossible, since $\exists$ never chooses green labels. So $G$ is acyclic in this case.

Now suppose instead that $\forall$ chooses $N^{\forall}\left(m_{1}, n_{2}\right)=T_{12}^{j}$ (some $j$ ), so $\Gamma^{+}=\Gamma \cup$ $\left\{m_{1}\right\}$. We claim that $j>0$. To see this, recall that we are supposing that there is at least one edge $\left(n_{1}, n_{2}\right)$ with $n_{1} \in \Gamma$ and $f_{\left(n_{1}, n_{2}\right)}=f^{i}$ for some $i>0$. By definition of $f_{\left(n_{1}, n_{2}\right)}$, this means that $\left(n_{1}, n_{2}\right)$ is linked to a tile edge $e$ in $N^{\forall}$ with tiling function $f^{i}$. However, as in Lemma $5(1)$, when the two edges chosen by $\forall$ in the current round are $N^{\forall}\left(m_{0}, n_{2}\right)=g_{02}$ and $N^{\forall}\left(m_{1}, n_{2}\right)=T_{12}^{j},\left(n_{1}, n_{2}\right)$ cannot be linked to an edge in $N$, so it must be linked to the edge $\left(m_{1}, n_{2}\right)$ and to no other edge of $N^{\forall}$. The tiling function for this edge is $f_{\left(m_{1}, n_{2}\right)}=f^{j}$. So $j=i>0$, as claimed.

Suppose, for contradiction, that there is a cycle $\gamma \subseteq G(|\gamma| \geq 3)$. Now, if $m_{1} \notin \gamma$, then we revert to the situation in the previous case: each node in $\gamma \cup\left\{m_{0}\right\}$ is incident with more than two $\forall$-edges within $\gamma \cup\left\{m_{0}\right\}$, which is impossible. So assume $m_{1} \in \gamma$. Which node of $\gamma \cup\left\{m_{0}\right\}$ was most recently added to the network? The most recent node should be incident with at most two edges in $\gamma \cup\left\{m_{0}\right\}$ chosen by $\forall$. As above, each node in $\Gamma \cap \gamma$ is incident with two edges within $\gamma$ labelled $\pm 1_{1}$ and one green edge connecting it to $m_{0}$, and all of these edges are only chosen by $\forall$. Therefore, either $m_{0}$ or $m_{1}$ was most recently added, and the edge $\left(m_{0}, m_{1}\right)$ must have been chosen by $\exists-$ otherwise, $m_{0}$ and $m_{1}$ are also incident with three edges chosen by $\forall$, since $m_{1} \in \gamma$.

So which atom would $\exists$ have chosen for the edge $\left(m_{0}, m_{1}\right)$ ? Her strategy for $(0-1)$ edges tells her to choose $u_{01}$ because of the existence of the cycle $\gamma$. But then, the current $\forall$-move is illegal, as $j>0$ and $g_{02} ; T_{21}^{j} \nsucceq u_{01}$ by rule 3 so that the triangle $\left(m_{0}, m_{1}, n_{2}\right)$ is inconsistent. This gives us a contradiction and proves the lemma.

This lemma allows us to define an integer valued $\operatorname{rank} r\left(n_{1}\right)$ for each node $n_{1}$ of $\Gamma^{+}$such that

$$
\text { if } n_{1}, n_{1}^{\prime} \in \Gamma^{+} \text {and } N\left(n_{1}, n_{1}^{\prime}\right)=+1_{1} \text {, then } r\left(n_{1}^{\prime}\right)=r\left(n_{1}\right)+1 \text {. }
$$

Now we can define co-ordinates for each new tile edge $\left(n_{1}, n_{2}\right)$ with $n_{1} \in \Gamma$ and $f_{\left(n_{1}, n_{2}\right)}=f^{i}$ for $i>0$.

Case A. Suppose that the two atoms chosen by $\forall$ are $N^{\forall}\left(m_{0}, n_{2}\right)=g_{02}$ and $N^{\forall}\left(m_{1}, n_{2}\right)=T_{12}^{j}$ (for some $m_{1} \in N$ and $j<k$ ). Here, $m_{1} \in \Gamma^{+}$; obviously, it is unique. We define co-ordinates for $\left(n_{1}, n_{2}\right)$ by

$$
\operatorname{Co}\left(n_{1}, n_{2}\right)=\left(r\left(n_{1}\right)-r\left(m_{1}\right), 0\right) .
$$


Case B. Suppose now that $\forall$ chooses the two edges $N^{\forall}\left(m_{0}, n_{2}\right)=g_{02}$ and $N^{\forall}\left(m_{2}, n_{2}\right)$ $=+1_{2}$ (the case where he chooses the atom $-1_{2}$ is similar). Again, $m_{2}$ is uniquely defined.

As $f\left(n_{1}, n_{2}\right) \neq f^{0},\left(n_{1}, n_{2}\right)$ must be linked to a tile edge of $N$. In fact, consideration of a shortest linking chain shows that it must be linked to one of the form $\left(n_{1}^{\prime}, m_{2}\right)$, for some $n_{1}^{\prime} \in \Gamma$, by a chain of attached tile edges of $M$ that are not edges of $N$. We have $f_{\left(n_{1}^{\prime}, m_{2}\right)}=f_{\left(n_{1}, n_{2}\right)} \neq f^{0}$, so $\left(n_{1}^{\prime}, m_{2}\right)$ has co-ordinates in $N$. Let $\operatorname{Co}\left(n_{1}^{\prime}, m_{2}\right)=(x, y)$, say. The co-ordinates of the new edge $\left(n_{1}, n_{2}\right)$ in $M$ are now defined to be

$$
\operatorname{Co}\left(n_{1}, n_{2}\right)=\left(x+r\left(n_{1}\right)-r\left(n_{1}^{\prime}\right), y+1\right) .
$$

(If $\forall$ 's second atom is $-1_{2}$, then replace $y+1$ by $y-1$.)

We have to show that this is well-defined. Suppose that $\left(n_{1}, n_{2}\right)$ is linked to two distinct such tile edges $\left(n_{1}^{\prime}, m_{2}\right)$ and $\left(n_{1}^{*}, m_{2}\right)$ by chains of the form stated. Then $\left(n_{1}^{\prime}, m_{2}\right)$ and $\left(n_{1}^{*}, m_{2}\right)$ are themselves linked in $M$ by such a chain - there is a chain of new tile edges

$$
\left(n_{1}^{\prime}, n_{2}\right)=\left(n_{1}^{1}, n_{2}\right),\left(n_{1}^{2}, n_{2}\right), \ldots,\left(n_{1}^{s}, n_{2}\right)=\left(n_{1}^{*}, n_{2}\right)
$$

of $M$, each successive pair being attached, with (evidently) $\left(n_{1}^{\prime}, m_{2}\right)$ attached to $\left(n_{1}^{\prime}, n_{2}\right)$ and $\left(n_{1}^{*}, n_{2}\right)$ attached to $\left(n_{1}^{*}, m_{2}\right)$. We have $n_{1}^{1}, \ldots, n_{1}^{s} \in \Gamma$. The proof of the claim in Lemma 5 (2) now applies, to show that $N\left(m_{0}, m_{2}\right)$ is green, the $\left(n_{1}^{i}, m_{2}\right)$ $(1 \leq i \leq s)$ are all tile edges of $N$, and that $\left(n_{1}^{i}, m_{2}\right)$ is attached to $\left(n_{1}^{i+1}, m_{2}\right)$ for each $i<s$. So $\left(n_{1}^{\prime}, m_{2}\right)=\left(n_{1}^{1}, m_{2}\right),\left(n_{1}^{2}, m_{2}\right), \ldots,\left(n_{1}^{s}, m_{2}\right)=\left(n_{1}^{*}, m_{2}\right)$ is a chain of attached tile edges of $N$.

Clearly, $n_{1}^{1}, n_{1}^{2}, \ldots, n_{1}^{s}$ is a path in $G$. So by T2 (in $N$ ) and the definition of the rank $r$, we have, for any $x, y \in \mathbb{Z}, C o\left(n_{1}^{i}, m_{2}\right)=\left(x+r\left(n_{1}^{i}\right), y\right) \Longleftrightarrow C o\left(n_{1}^{i+1}, m_{2}\right)=$ $\left(x+r\left(n_{1}^{i+1}\right), y\right)$ for each $i<s$. We now obtain

(*) if $\operatorname{Co}\left(n_{1}^{\prime}, m_{2}\right)=(x, y)$, then $\operatorname{Co}\left(n_{1}^{*}, m_{2}\right)=\left(x+r\left(n_{1}^{*}\right)-r\left(n_{1}^{\prime}\right), y\right)$,

by induction on $s$. Thus, the co-ordinate function is well-defined.

We have now defined tilings for all tile edges of $M$ that are not in $N$, and co-ordinates for those whose associated tiling is not $f^{0}$.

6.6. Conditions T1, T2 hold for $M$. Let us now check that $M$ satisfies conditions $\mathrm{T} 1$ and $\mathrm{T} 2$. It is sufficient to check that if the tile edges $e, e^{\prime}$ of $M$ are attached, then they share a tiling function and, if appropriate, their co-ordinates match according to T2. Since T1 and T2 hold for $N$, we can assume that $e, e^{\prime}$ are not both edges of $N$.

It follows that $e, e^{\prime}$ are not both edges of $N^{\forall}$. For if they were, then being attached, they form two sides of a triangle $\Delta$ which is not a $\forall$-triangle, the third side of $\Delta$ being labelled by a \pm 1 atom. Because $\exists$ never chose a \pm 1 label in this (or any) round, the third side of $\Delta$ is also labelled in $N^{\forall}$. So all three sides of $\Delta$ are labelled in $N^{\forall}$. But by the preceding paragraph, $\Delta$ does not lie within $N$, so $\Delta$ must in fact be $\forall$ 's triangle in the current round. This is a contradiction.

So we may assume that $e$ is not an edge of $N^{\forall}$. This means that $e$ is a tile edge labelled by $\exists$ in the current round. Say, $e=\left(n_{1}, n_{2}\right)$ for some $n_{1} \in N$.

Case 1: Assume that $e^{\prime}$ is an edge of $N$. So $e^{\prime}$ must have the form $\left(n_{1}, m_{2}\right)$ where $m_{2} \in N$ and $M\left(m_{2}, n_{2}\right)= \pm 1_{2}$. Then as $e, e^{\prime}$ are certainly linked in $M$, by the well-definedness of the tiling function we have $f_{(e)}=f_{\left(e^{\prime}\right)}$, so $\mathrm{T} 1$ holds for $e, e^{\prime}$. Moreover, if $f_{(e)} \neq f^{0}$, then by the well-definedness 
of the co-ordinates, if $C o\left(e^{\prime}\right)=(x, y)$ and $M\left(m_{2}, n_{2}\right)=+1_{2}$, say, we have $C o(e)=(x, y+1)$. Thus, the condition in $\mathrm{T} 2$ is met. The case where $M\left(m_{2}, n_{2}\right)=-1_{2}$ is similar.

Case 2: Assume that $e^{\prime}$ is an edge of $N^{\forall}$ but not an edge of $N$. Thus, it was chosen by $\forall$ in the current round, and has the form $\left(m_{1}, n_{2}\right)$, where $N\left(m_{1}, n_{1}\right)= \pm 1_{1}$. Its co-ordinates, if any, are $(0,0)$. As in the previous case, $f_{(e)}=f_{\left(e^{\prime}\right)}$, so T1 is satisfied. If $f_{(e)} \neq f^{0}$ and $N\left(m_{1}, n_{1}\right)=+1_{1}$, say, then again by definition of the co-ordinates we have $C o(e)=(1,0)$. If $N\left(m_{1}, n_{1}\right)=-1_{1}, C o(e)=(-1,0)$. Hence, T2 is met.

Case 3: Assume finally that $e, e^{\prime}$ are both edges of $M$ that are not in $N^{\forall}$. Then they are linked in $M$. As 'linked' is an equivalence relation, if $e$ is linked to a tile edge $e^{*}$ of $N^{\forall}$, then so is $e^{\prime}$, and we have $f_{(e)}=f_{\left(e^{\prime}\right)}=f_{\left(e^{*}\right)}$. If $e$ is not linked to any tile edge of $N^{\forall}$, then neither is $e^{\prime}$ and we have $f_{(e)}=f_{\left(e^{\prime}\right)}=f^{0}$. Hence $f_{(e)}=f_{\left(e^{\prime}\right)}$ in any case, and T1 is met. Let $e^{\prime}=\left(n_{1}^{\prime}, n_{2}\right)$, and assume that $N\left(n_{1}, n_{1}^{\prime}\right)=+1_{1}$ (the case $N\left(n_{1}, n_{1}^{\prime}\right)=-1_{1}$ is similar). If $f_{(e)} \neq f^{0}$ then $e, e^{\prime}$ are assigned co-ordinates as above, and there are two ways this can happen.

Case 3a: Suppose first that $e$ is linked in $M$ to a tile edge $e^{*}=\left(m_{1}, n_{2}\right)$ chosen by $\forall$ in the current round. (So $\forall$ 's atoms were $N^{\forall}\left(e^{*}\right)$ and the green atom forcing the existence of the tile edges $e, e^{\prime}$.) Then $e^{\prime}$ is also linked to $e^{*}$, and we have

$$
\begin{aligned}
C o(e) & =\left(r\left(n_{1}\right)-r\left(m_{1}\right), 0\right) \\
C o\left(e^{\prime}\right) & =\left(r\left(n_{1}^{\prime}\right)-r\left(m_{1}\right), 0\right) .
\end{aligned}
$$

As $N\left(n_{1}, n_{1}^{\prime}\right)=+1_{1}$, the rank $r$ satisfies $r\left(n_{1}^{\prime}\right)=r\left(n_{1}\right)+1$, and this yields that $C o(e)$ and $C o\left(e^{\prime}\right)$ are in accordance with T2.

Case 3b: Suppose now that $e$ is linked in $M$ to a tile edge $e^{*}$ of $N$ by a chain of attached tile edges of $M$ that are not in $N$. Then so is $e^{\prime}$ (by extending the chain by the extra link $\left.\left(e^{\prime}, e\right)\right)$. We saw that $e^{*}$ can be taken to be of the form $\left(n_{1}^{*}, m_{2}\right)$, where $N^{\forall}\left(m_{2}, n_{2}\right)= \pm 1_{2}$ - say, $-1_{2}$. Let $\operatorname{Co}\left(e^{*}\right)=(x, y)$. Then the definition of the new co-ordinates (above) gives

$$
\begin{aligned}
C o(e) & =\left(x+r\left(n_{1}\right)-r\left(n_{1}^{*}\right), y-1\right) \\
C o\left(e^{\prime}\right) & =\left(x+r\left(n_{1}^{\prime}\right)-r\left(n_{1}^{*}\right), y-1\right) .
\end{aligned}
$$

From the definition of the rank $r, r\left(n_{1}^{\prime}\right)=r\left(n_{1}\right)+1$, so that if $C o(e)=(z, t)$ then, $\operatorname{Co}\left(e^{\prime}\right)=(z+1, t)$, in accordance with $\mathrm{T} 2$.

6.7. Tile atoms for the new edges, and consistency. Now we have a tiling function and (if necessary) co-ordinates for each new tile edge to be labelled by $\exists$. For each such edge $e, \exists$ lets

$$
M(e)= \begin{cases}T_{12}^{0}, & \text { if } f_{(e)}=f^{0} \\ T_{12}^{f_{(e)}(C o(e))}, & \text { otherwise }\end{cases}
$$

in accordance with T3. It remains to check that triangles involving these edges are consistent. There are two rules involving tile edges: rules 3 and 2

For rule [3, suppose for a contradiction that the new tile edge $\left(n_{1}, n_{2}\right)$ lies in a triangle $\Delta$ in $M$ whose other sides are labelled by $g_{01}$ and $u_{02}$, or alternatively $g_{02}$ and $u_{01}$. There can only be a problem with rule[3 if $M\left(n_{1}, n_{2}\right)=T_{12}^{i}$ for some $i$ with $i>0$. Now $\exists$ only chooses $T_{12}^{i}$ with $i>0$ if $\left(n_{1}, n_{2}\right)$ is linked to a tile edge 
of $N^{\forall}$. That means that in the current round, $\forall$ must have chosen one green atom $g_{02}$ and either $\pm 1_{2}$ or a tile atom $T_{12}^{j}$ (some $j$ ). All other edges incident with $n_{2}$ were chosen by $\exists$. The edge $\left(m_{0}, n_{2}\right)$ (say) of $N^{\forall}$ labelled $g_{02}$ is not in $\Delta$, since we know that $N\left(m_{0}, n_{1}\right)=g_{01}$ (else $\exists$ would not choose a tile to label $\left(n_{1}, n_{2}\right)$ ). So it follows that $\exists$ must have chosen a second side of the triangle $\Delta$ as well as $\left(n_{1}, n_{2}\right) . \exists$ never chooses a green atom, so this second side must be labelled with the $u$-atom. But we have already checked (when we considered her strategy for $(0-1)$ and $(0-2)$ edges $)$ that no triangles involving $u$-labelled edges chosen by $\exists$ are inconsistent. Thus, rule 3 cannot be violated.

That leaves the crucial rule 2. Suppose $\exists$ chooses $M\left(n_{1}, n_{2}\right)=T_{12}^{i}$, and this edge is part of a triangle $\left(n_{1}, n_{2}, n_{1}^{\prime}\right)$ say, with $M\left(n_{1}^{\prime}, n_{2}\right)=T_{12}^{j}$ and $M\left(n_{1}^{\prime}, n_{1}\right)=+1_{1}$, say (the other three cases, in which +1 is replaced by -1 and/or $n_{1}^{\prime}$ is replaced by a 2-node $n_{2}^{\prime}$, are entirely similar).

This triangle $\left(n_{1}, n_{2}, n_{1}^{\prime}\right)$ is not an $\forall$-triangle, because if it were it would be in $N^{\forall}$, whereas in fact one of its edges is a tile edge labelled by $\exists$. Hence the two tile edges in it are attached. By T1, they share a tiling function, say $f^{l}$. If $l=0$, then by T3 we have $i=j=0$, so there is no problem with rule2. If $l>0$, by T2 we have $C o\left(n_{1}^{\prime}, n_{2}\right)=(x, y)$ and $C o\left(n_{1}, n_{2}\right)=(x+1, y)$ for some $x, y \in \mathbb{Z}$. By T3, $f^{l}(x, y)=j$ and $f^{l}(x+1, y)=i$. Because $f^{l}$ is a valid tiling, $\operatorname{Lt}\left(T_{i}\right)=\operatorname{Rt}\left(T_{j}\right)$. Hence the labels on the triangle $\left(n_{1}, n_{2}, n_{1}^{\prime}\right)$, which are $\left(T_{12}^{i}, T_{21}^{j},+1_{1}\right)$, are consistent with rule 2.

Thus triangles involving new edges are consistent with all the rules.

This completes $\exists$ 's strategy. In each case she is able to choose an atom without creating any inconsistent triangles and she is able to define coherent tilings and co-ordinates where appropriate. In this way she can continue forever and win the game $G(R A(\tau))$.

\section{Associativity AND CONCLUSION}

Lemma 7. Suppose that $\exists$ has a winning strategy in the game $G(R A(\tau))$. Then $R A(\tau)$ is associative.

Proof. This is well-known. Let $r, s, t \in R A(\tau)$. We show that $(r ; s) ; t \leq r ;(s ; t)$. Take an atom $x \leq(r ; s) ; t$. Because for any $p, q \in R A(\tau)$ we have

$$
p ; q=\sum\{a ; b: a, b \text { atoms of } R A(\tau), a \leq p, b \leq q\},
$$

we see that there are atoms $y \leq r ; s$ and $c \leq t$ with $x \leq y$; $c$. Similarly, there are atoms $a \leq r, b \leq s$ with $y \leq a ; b$.

Let $\forall$ begin a play of $G(R A(\tau))$ with the atom $y$. Using her winning strategy in this game, $\exists$ will respond with a network $N_{0}$ with nodes 1,3 , say, not necessarily distinct, with $N_{0}(1,3)=y$. Let $\forall$ continue by picking the edge $(1,3)$ and atoms $a, b$, and then the edge $(1,3)$ again and atoms $x, \breve{c}$. (We suspend Convention 1(2), to allow $\forall$ to make any consistent moves he likes. This is quite harmless.) $\exists$ responds after these two moves with a network $N_{2}=N$ with nodes $1,2,3,4$ such that $N(1,2)=a, N(2,3)=b, N(3,4)=c$, and $N(1,4)=x$. See Figure 8 .

Let $N(2,4)=z$. Then because $N$ is a network and $z$ an atom, we have $z \leq b ; c$, so that $z \leq s ; t$, and $x \leq a ; z$, so that $x \leq r ;(s ; t)$. Because the atom $x \leq(r ; s) ; t$ was arbitrary, and $R A(\tau)$ is atomic, we have $(r ; s) ; t \leq r ;(s ; t)$.

A similar argument shows that $(r ; s) ; t \geq r ;(s ; t)$. This proves associativity. 


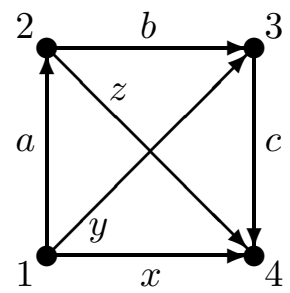

Figure 8. Associativity of $R A(\tau)$

Assume that $\tau$ is a yes-instance of the tiling problem. From Theorem 4 we get that $\exists$ has a winning strategy in $G(R A(\tau))$. By Lemma 7, $R A(\tau)$ is associative, so by Theorem 1, it is representable. Now assume that $\tau$ is a no-instance. Then either $R A(\tau)$ is not associative (and this is decidable), or else it is, in which case by Theorems 1 and 3, $R A(\tau)$ is not representable. So we obtain our result:

Theorem 8. The problem of deciding whether a finite relation algebra is representable or not is undecidable.

Corollary 9. The set of isomorphism types of representable finite relation algebras possessing only infinite representations is not recursively enumerable.

For a proof, see the discussion in the introduction.

\section{AdDED AFTER POSTING}

There is an error in the proof of part 2 of Lemma 5 of the originally posted article. In the proof of the claim that $N\left(m_{0}, m_{2}\right)=g_{02}$, the statement that "If $\exists$ chose the edge $\left(m_{0}, m_{2}\right)$ then, by her strategy for $(1-2)$ edges and because of the prior existence of the chain $C$, she would have chosen $N\left(m_{0}, m_{2}\right)=v_{02}$ " is not correct, as we do not know that the tile edges $\left(m_{2}, n_{1}^{1}\right),\left(m_{2}, n_{1}^{s}\right)$ are labelled in $N^{\forall}$ in the round when $\exists$ labels $\left(m_{0}, m_{2}\right)$, as required by $\exists$ 's strategy.

We therefore alter the definition of $\exists$ 's strategy by dropping the requirement that these edges are labelled in $N^{\forall}$. Now, we only require that they be tile edges. There are consequential amendments to the checks that the strategy is well-defined and consistent. The corrections are listed below; they affect only the page or so of $\S 6.1$ between II and III.

We thank Ágnes Kurucz for useful comments on correcting the mistake.

Definition of $\exists$ 's strategy. After the definition of condition $(\boldsymbol{\beta})$, insert:

"Remark A. Clearly, $(\boldsymbol{\beta})$ completely determines which edges of $M$ will be tile edges and which will not: an edge of $M$ will be a tile edge iff either it is already labelled in $N^{\forall}$ with a tile atom, or it forms one side of a triangle in $M$ whose other two edges are labelled $g_{01}, g_{02}$ in $N^{\forall}$."

In $\exists$ 's strategy, case $\mathbf{I I}$, replace condition $2(c), " N^{\forall}\left(n_{1}, c_{2}\right)=T_{12}^{i}$ and $N^{\forall}\left(n_{1}, d_{2}\right)$ $=T_{12}^{j}$ (some $\left.i, j<k\right)$," by the following:

" $\left(n_{1}, c_{2}\right)$ and $\left(n_{1}, d_{2}\right)$ will be tile edges of $M$ (cf. Remark A above)," 
Check that the strategy is well-defined. Replace the 5 lines "But both $n_{0}$ and $n_{1}$ are incident with at least four such edges ... strategy is well-defined" by the following:

"But $n_{0}$ is incident with four such edges, because $N^{\forall}\left(n_{0}, g\right)=g_{01}\left(g \in \gamma \backslash\left\{n_{1}\right\}\right)$ and $N^{\forall}\left(n_{0}, \alpha_{2}\right)=g_{02}\left(\alpha_{2} \in C\right)$. So the node being currently added must be $n_{1}$. The two edges connecting $n_{1}$ to its neighbours in $\gamma$ are labelled with $\pm 1_{1}$ in $N^{\forall}$, so by $(\boldsymbol{\alpha})$, these must be the two edges labelled by $\forall$ in the current round - in particular, he chose no green atoms. By $(\boldsymbol{\beta}), \exists$ does not choose any tile atoms in this round, so the tile edges $\left(n_{1}, c_{2}\right),\left(n_{1}, d_{2}\right)$ must also be labelled by $\forall$ in the current round. This contradicts the fact that $n_{1}$ is incident with only two labelled edges in $N^{\forall}$, and shows that the strategy is well-defined."

Check that the strategy is consistent. Replace the 9 lines "Suppose there is a chain $C$ with endpoints $c_{2} \neq d_{2} \ldots$ Thus, the situation described does not arise." by the following:

"Suppose there is a chain $C$ with endpoints $c_{2} \neq d_{2}$, with $\left(n_{1}, c_{2}\right)$ and $\left(n_{1}, d_{2}\right)$ both tile edges of $M$, as in case 2 of the strategy, and with $M\left(n_{0}, m_{1}\right)=g_{01}$ and $M\left(m_{1}, n_{1}\right)= \pm 1_{1}$. As before, because the labels $g_{02}$ on the edges $\left(n_{0}, c_{2}\right)$ and $\left(n_{0}, d_{2}\right)$ must have been chosen by $\forall$, this could only happen if $n_{1}$ is the node currently added, as $c_{2} \neq d_{2}$.

Since by $(\boldsymbol{\alpha})$ the edge $\left(n_{1}, m_{1}\right)$ is labelled in $N^{\forall}$, at most one of the two tile edges $\left(n_{1}, c_{2}\right),\left(n_{1}, d_{2}\right)$ can also be labelled in $N^{\forall}$, so $\exists$ is going to label at least one of them with a tile atom. So by $(\boldsymbol{\beta}), \forall$ must have chosen a green atom in the current round. His choices of atoms and nodes were therefore $\pm 1_{1}, g_{01}$, and $m_{1}, p_{0}$, say, for some $p_{0} \in N: N^{\forall}\left(p_{0}, n_{1}\right)=g_{01}$. Hence, $\exists$ labelled both tile edges $\left(n_{1}, c_{2}\right)$, $\left(n_{1}, d_{2}\right)$. By $(\boldsymbol{\alpha}),\left(p_{0}, n_{1}\right)$ is the only green edge incident with $n_{1}$; so by $(\boldsymbol{\beta})$, we must have $N\left(p_{0}, c_{2}\right)=N\left(p_{0}, d_{2}\right)=g_{02}$. Note that $p_{0} \neq n_{0}$, since $M\left(n_{1}, n_{0}\right)$ is not green.

But now we consider which node from $C \cup\left\{n_{0}, p_{0}\right\}$ was most recently added, as the game progressed through earlier rounds. Whichever it is, it can be connected to the rest of $C \cup\left\{n_{0}, p_{0}\right\}$ by at most two edges labelled by $\forall$. Nodes in $C \backslash\left\{c_{2}, d_{2}\right\}$ are incident with three edges chosen by $\forall$ - two edges within $C$ labelled $\pm 1_{2}$, and one to $n_{0}$, labelled $g_{20}$ - so it was not any of those (if there are any). Nor was it $c_{2}$ or $d_{2}$, as they are each incident with a $\pm 1_{2}$ edge (within $C$ ) and two green edges, to $p_{0}$ and $n_{0}$. So the most recently added node must have been either $n_{0}$ or $p_{0}$. These are both connected to $c_{2}$ and $d_{2}$ by edges labelled by $g_{02}$. Because $c_{2} \neq d_{2}$, we see that in the round when the last of $n_{0}, p_{0}$ was built, $\forall$ must have selected the nodes $c_{2}, d_{2}$ and the atoms $g_{20}, g_{02}$. But this is in contravention to convention 1 , since there was a suitable node (namely whichever of $n_{0}, p_{0}$ already existed) in the network already, so $\forall$ should not have made such a move. This contradiction shows that if $\exists$ chooses $M\left(n_{0}, n_{1}\right)=v_{01}$ then any triangle involving this edge is consistent."

Clarification: edges of $N^{\forall}$. On a separate matter, in later parts of the paper the term "edge of $N^{\forall}$ " is used over-loosely to mean "labelled edge of $N^{\forall}$ ". For example, in the first sentence of $\S 6.5$, the phrase " ... which is not an edge of $N^{\forall}$ ..." means "... which is not a labelled edge of $N^{\forall} \ldots$ " In the third sentence before the end of the second paragraph in the proof of Lemma 6, the phrase "... and to no other edge of $N^{\forall}$." means "... and to no other labelled edge of $N^{\forall}$.". The first sentence of the second paragraph of $\S 6.6$, "It follows that $e, e^{\prime}$ are not both edges of $N^{\forall}$." means "It follows that $e, e^{\prime}$ are not both labelled edges of $N^{\forall}$.". The 
first sentence of the third paragraph of $\S 6.6$, "So we may assume that $e$ is not an edge of $N^{\forall}$.", means "So we may assume that $e$ is not a labelled edge of $N^{\forall}$.". In the first sentence of Case 2, the phrase " $\ldots e^{\prime}$ is an edge of $N^{\forall} \ldots$ " means "... $e^{\prime}$ is a labelled edge of $N^{\forall} \ldots$ ". In the first sentence of Case 3, the phrase "... edges of $M$ that are not in $N^{\forall}$." means "... edges of $M$ that are not labelled in $N^{\forall} . "$.

\section{REFERENCES}

[AMN91] H. Andréka, J. D. Monk, and I. Németi. Algebraic Logic. Colloq. Math. Soc. J. Bolyai. North-Holland, Amsterdam, 1991. Conference Proceedings, Budapest, 1988. MR 92m:03003

[Ber66] R. Berger. The undecidability of the domino problem. Memoirs of the American Mathematical Society, vol. 66, 1966. MR 36:49]

[HH97a] R. Hirsch and I. Hodkinson. Complete representations in algebraic logic. Journal of Symbolic Logic, 1997. 62(3):816-847, 1997. MR 98m:03123

[HH97b] R. Hirsch and I. Hodkinson. Step by step - building representations in algebraic logic. Journal of Symbolic Logic. 62(1):225-279, 1997. MR 98g:03145

[Hir95] R. Hirsch. Completely representable relation algebras. Bulletin of the Interest Group in Propositional and Predicate Logics, 3(1):77-91, 1995. MR 96d:03082

[HMT85] L. Henkin, J. D. Monk, and A. Tarski. Cylindric Algebras. Part II. North-Holland, 1985. MR 86m:03095b

[Hod97] I. Hodkinson. Atom structures of cylindric algebras and relation algebras. Ann. Pure Appl. Logic, 89(2-3):117-148, 1997. CMP 98:06

[JT48] B. Jónsson and A. Tarski. Representation problems for relation algebras. Bulletin of the American Mathematical Society, 54, pp. 80 and 1192, 1948.

[JT52] B. Jónsson and A. Tarski. Boolean algebras with operators. II. American Journal of Mathematics, 74:127 - 162, 1952. MR 13:524g

[Lyn50] R. Lyndon. The representation of relational algebras. Annals of Mathematics, 51(3):707-729, 1950. MR 12:237a

[Lyn56] R. Lyndon. The representation of relation algebras, II. Annals of Mathematics, 63(2):294-307, 1956. MR 18:106f

[Ma82] R. Maddux. Some varieties containing relation algebras. Transactions of the American Mathematical Society, 272(2):501-526, 1982. MR 84a:03079

[Ma91a] R. Maddux. Introductory course on relation algebras, finite-dimensional cylindric algebras, and their interconnections. In AMN91, pages 361-392. MR 93c:03082

[Ma91b] R. Maddux. The origin of relation algebras in the development and axiomatization of the calculus of relations. Studia Logica, 5(3-4):421-456, 1991. MR 93c:03082

[Ma94] R. Maddux. A perspective on the theory of relation algebras. Algebra Universalis, 31:456-465, 1994. MR 95c:03143

[Mon64] J. D. Monk. On representable relation algebras. Michigan Mathematics Journal, 11:207210, 1964. MR 30:3016

[Nem96] I. Németi. A fine-structure analysis of first-order logic. In M. Marx, L. Pólos, and M. Masuch, editors, Arrow Logic and Multi-Modal Logic, Studies in Logic, Language and Information, pages 221-247. CSLI Publications \& FoLLI, 1996. CMP 97:09

[TG87] A. Tarski and S. R. Givant. A Formalization of Set Theory Without Variables. Number 41 in Colloquium Publications in Mathematics. American Mathematical Society, Providence, Rhode Island, 1987. MR 89g:03012

Department of Computer Science, University College, Gower Street, London, WC1E 6BT, U.K.

E-mail address: r.hirsch@cs.ucl.ac.uk

URL: http://www.cs.ucl.ac.uk/staff/R.Hirsch/

Department of Computing, Imperial College, Queen's Gate, London SW7 2BZ, U.K.

E-mail address: imh@doc.ic.ac.uk

URL: http://www.doc.ic.ac.uk/ imh/ 